\title{
THE ROLE OF STREAMERS IN THE DEFLECTION OF CORONAL MASS EJECTIONS: COMPARISON BETWEEN STEREO THREE-DIMENSIONAL RECONSTRUCTIONS AND NUMERICAL SIMULATIONS
}

\author{
F. P. Zuccarello ${ }^{1,2}$, A. Bemporad ${ }^{3}$, C. Jacobs ${ }^{1}$, M. Mierla ${ }^{4,5,6}$, S. Poedts $^{1}$, and F. Zuccarello ${ }^{7}$ \\ ${ }^{1}$ Centre for Plasma-Astrophysics, K. U. Leuven, Celestijnenlaan 200B, 3001 Leuven, Belgium; Francesco.Zuccarello@wis.kuleuven.be, \\ Carla.Jacobs@wis.kuleuven.be, Stefaan.Poedts@wis.kuleuven.be \\ 2 INAF-Osservatorio Astrofisico di Catania, via S. Sofia 78, 95123 Catania, Italy \\ ${ }^{3}$ Istituto Nazionale di Astrofisica (INAF), Osservatorio Astronomico di Torino, Strada Osservatorio 20, 10025 Pino Torinese, Torino, Italy; \\ bemporad@oato.inaf.it \\ ${ }^{4}$ Royal Observatory of Belgium, Brussels, Belgium; marilena@oma.be \\ 5 Institute of Geodynamics of the Romanian Academy, Bucharest, Romania \\ ${ }^{6}$ Research Center for Atomic Physics and Astrophysics, Faculty of Physics, University of Bucharest, Romania \\ ${ }^{7}$ Dipartimento di Fisica e Astronomia, Universitá di Catania, Via S. Sofia 78, 95123 Catania, Italy; fzu@oact.inaf.it \\ Received 2011 July 27; accepted 2011 September 8; published 2011 December 14
}

\begin{abstract}
On 2009 September 21, a filament eruption and the associated coronal mass ejection (CME) were observed by the Solar Terrestrial Relations Observatory (STEREO) spacecraft. The CME originated from the southern hemisphere and showed a deflection of about $15^{\circ}$ toward the heliospheric current sheet (HCS) during the propagation in the COR1 field of view. The CME source region was near the central meridian, but no on-disk CME signatures could be seen from the Earth. The aim of this paper is to provide a physical explanation for the strong deflection of the CME observed on 2009 September 21. The two-sided view of the STEREO spacecraft allows us to reconstruct the three-dimensional travel path of the CME and the evolution of the CME source region. The observations are combined with a magnetohydrodynamic simulation, starting from a magnetic field configuration closely resembling the extrapolated potential field for that date. By applying localized shearing motions, a CME is initiated in the simulation, showing a similar non-radial evolution, structure, and velocity as the observed event. The CME gets deflected toward the current sheet of the larger northern helmet streamer due to an imbalance in the magnetic pressure and tension forces and finally gets into the streamer. This study shows that during solar minima, even CMEs originating from high latitude can be easily deflected toward the HCS, eventually resulting in geoeffective events. How rapidly they undergo this latitudinal migration depends on the strength of both the large-scale coronal magnetic field and the magnetic flux of the erupting filament.
\end{abstract}

Key words: magnetohydrodynamics (MHD) - methods: numerical - methods: observational - Sun: corona - Sun: coronal mass ejections (CMEs)

Online-only material: color figures

\section{INTRODUCTION}

Understanding the dynamics of solar phenomena such as flares and coronal mass ejections (CMEs) requires joint observational and theoretical research efforts. Detailed event studies, combining observations and simulations, yield a better insight into CME dynamics and its propagation throughout the interplanetary (IP) medium. One of the most studied events is the series of eruptions that, during the end of 2003 October, led to a series of geomagnetic storms, known as the Halloween storms. Manchester et al. (2008) performed a three-dimensional (3D) magnetohydrodynamic (MHD) simulation of the eruptions occurring on 2003 October 26 and 28 using a data-driven model for the background solar corona. The synthetic whitelight images produced from their numerical simulation are qualitatively in agreement with the observed Large Angle and Spectrometric Coronagraph Experiment (LASCO) C2 and C3 images. They concluded that the appearance of the CME is largely determined by the density structure of the pre-event solar corona and may not be sensitive to the initiation process. Using a 3D kinematic model, Intriligator et al. (2005) investigated the propagation of the 2003 October/November eruptions to a distance of more than $90 \mathrm{AU}$. Their results indicate the importance of considering longitudinal and latitudinal variations in the propagation of CMEs in order to explain spacecraft measurements. Another well-studied event is the 1997 May 12 halo CME. Using the ambient solar wind derived from coronal models and utilizing photospheric magnetic field observations, Odstrcil et al. (2005) performed a numerical simulation of this event. Using a simple "ice-cream cone" model for the CME (Zhao et al. 2002), the authors were able to predict the arrival of the shock and of the ejecta. The heliospheric evolution and interaction of the two CMEs that occurred during 2007 January 24-27 were investigated by Lugaz et al. (2009). By combining both multi-viewpoint observations with 3D MHD simulations, the 3D structure of the CMEs could be unraveled. The synthetic line-of-sight (LOS) images successfully reproduced the LASCO observations, showing the deflection of the helmet streamers due to the interaction with the first CME, and the flattening of the second CME as it propagates in the deflected streamer. Rušin et al. (2010) compared observations of the solar corona during the eclipse of 2008 August 1 with MHD simulations of the steady corona (Lionello et al. 2009). By including complex physical processes as thermal conduction along the field lines, radiative losses, Alfvén wave pressure, and a parameterized heating term, the large-scale structure of the corona was well reproduced. The authors also noticed that even though there were no active regions (ARs) on the solar disk, the corona presented a quite complex streamer configuration.

The relationship between CMEs and streamers has been investigated by Subramanian et al. (1999) using the LASCO data up to six solar radii acquired during the solar minimum 
period of cycle 22, i.e., from 1996 January to 1998 June. They found that $63 \%$ of the investigated events could be connected to coronal streamers. In about one-third of the streamer-related events, the CME had disrupted the streamer structure. The extreme solar minimum of the previous solar cycle is an excellent opportunity to study the relation between the global coronal field and CMEs, as was done by Liu et al. (2009). By combining multi-viewpoint observations with a potentialfield source-surface (PFSS) reconstruction of the magnetic field, these authors investigated the coronal structure after the 2007 December $31 \mathrm{CME}$. The helmet streamer, disrupted by the CME, reforms, but is shifted southward and the southern coronal hole shrinks considerably. This points out that CMEs might play a considerable role in the evolution of the large-scale coronal magnetic field. On the other hand, in such solar minimum conditions, the interaction between the global coronal magnetic field and the CME can also result in the deflection of the CME without affecting the large-scale magnetic structure.

Since the Skylab and Solar Maximum Mission era (e.g., MacQueen et al. 1986), the occurrence of latitudinal deflections of CMEs toward the equator is a well-known phenomenon, as well as similar deflections of flare-associated shock waves (e.g., Fengsi \& Dryer 1991). The proposed explanation at that time was that the observed equatorward deflections were due to the background large-scale bipolar field for CMEs, responsible for non-radial forces affecting the CME trajectories (MacQueen et al. 1986), and to the heliospheric magnetic field for IP shocks (Wei \& Dryer 1991). Later on, many detailed investigations of deflections were performed in the Solar and Heliospheric Observatory (SOHO) era: statistical results show that during solar minima, CME deflections occur preferentially toward the equator, while during periods of intense solar activity deflection both toward the equator and toward the poles is observed, depending on the location and total area of coronal holes (Cremades et al. 2006). These results suggested a second possible explanation for the observed deflections, i.e., that due to the faster Alfvén velocities in coronal holes, the shock driven by the CME could be reflected, such that coronal holes would act as a "magnetic wall" that constrains the CME propagation (Gopalswamy et al. 2009). CME deflections became a very popular research topic in the present Solar Terrestrial Relations Observatory (STEREO) era, obviously because stereoscopic observations provided by the twin STEREO spacecraft today allow a determination of the 3D trajectory of CMEs, with many different techniques (see Mierla et al. 2010, for a recent review). Recently, deflections of CMEs and interplanetary CMEs measured with STEREO 3D reconstruction techniques have been reported by many authors (e.g., Kilpua et al. 2009; Byrne et al. 2010; Liewer et al. 2010) and measurements of not only latitudinal but also longitudinal CME deflections are now possible (Liu et al. 2010a, 2010b; Lugaz et al. 2010). It is also well known that coronal streamers may act as CME deflectors. "Streamer puffs" (Bemporad et al. 2005; Moore \& Sterling 2007; Jiang et al. 2009), for instance, are a sub-class of CMEs originating from compact ejective flares in an outer flank of the base of a streamer, resulting in a small-scale eruption laterally deflected and channeled by the streamer fields. This "channeling" effect by coronal streamers has also been simulated by Filippov et al. (2001) for eruptive solar filaments. A theoretical explanation for CME deflections measured with STEREO/COR1 and COR2 data has been recently proposed by Shen et al. (2011): as the CME expands and compresses the surrounding corona, the additional free magnetic energy from compression provides a restoring force that acts on the CME. Since the background magnetic field is not uniformly distributed around the CME, a resultant force will push the CME toward the lower magnetic energy density region, thus explaining the equatorward CME deflections at solar minimum. Nevertheless, the process of interaction between CME expansion and the surrounding coronal structures needs to be investigated in more detail to understand the underlying physics more precisely. Neighboring coronal holes can play a role in the CME deflections, but other factors are certainly involved as well.

The aim of this paper is to provide a physical explanation for the deflection of the CME observed on 2009 September 21. In order to do this, we use STEREO/SECCHI observations to reconstruct the $3 \mathrm{D}$ dynamics of the $\mathrm{CME}$ and combine these with MHD simulations of this event. Finally, we compare the results of simulations with the observations in order to understand the role that the global coronal field played in the CME deflection. In the next section, the morphological evolution of the CME, its $3 \mathrm{D}$ reconstruction, and the photospheric dynamics that preceded the event are described. The initial configuration, the boundary conditions, and the results of the numerical MHD simulations are presented in Section 3, while the comparison between simulations and observations is discussed in Section 4. The summary of our work is presented in Section 5.

\section{OBSERVATIONS}

\subsection{General Morphology Deduced from STEREO and SOHO}

On 2009 September 21, a small prominence eruption leading to a CME occurred. This eruption was observed by both the SECCHI-Extreme UV Imagers (EUVI) and the COR1 coronagraphs aboard the twin STEREO (see Kaiser et al. 2008) spacecraft. The two identical EUVI instruments (Wuelser et al. 2004) observed the chromosphere and corona with four different bandpass filters, centered on the He II $304 \AA$, Fe IX $171 \AA$, Fe XII $195 \AA$, and Fe XV $284 \AA$ emission lines, with bandwidths between 14 and $30 \AA$. Response curves peak at temperatures around $T=10^{4.9} \mathrm{~K}, T=10^{6.0} \mathrm{~K}, T=10^{6.2} \mathrm{~K}$, and $T=10^{6.3} \mathrm{~K}$, respectively, but emission from plasma at larger or smaller temperatures may also be important, in particular for the He II and Fe XV filters with much broader bandpass distributions. Observations were acquired with back-illuminated CCDs with $2048 \times 2048$ pixels. The typical field of view (FOV) extends up to $\sim 1.7 R_{\odot}$, with a spatial resolution around $1.6 \operatorname{arcsec}_{\text {pixel }}{ }^{-1}$.

On the day of the eruption, the two EUVI-A and EUVI-B instruments were observing with spatial resolutions of

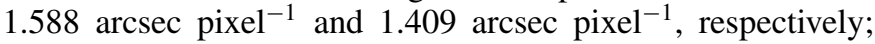
this small difference is due to the different distance of the STEREO-A and STEREO-B spacecraft from the Sun. Data with the He II filter were acquired with a temporal resolution of 10 minutes, while data with other filters were acquired with much lower cadence. The initiation of this eruption was well observed by STEREO-B in EUVI He II $304 \AA$ as a thin plasma "tongue" leaving the Sun from the southwest limb (Figure 1, top panels). In STEREO-B images, the prominence material starts to rise in altitude very early, around 11:00 UT, leaving the Sun from an apparent projected latitude of $\simeq 37^{\circ}$ south. In the early phases of the eruption, the prominence plasma expands mainly in the radial direction, forming the "tongue" of material shown in Figure 1 (top panels). Later on, around 15:00 UT a significant counterclockwise rotation of the prominence occurred during the expansion, resulting in a northward propagation. This 

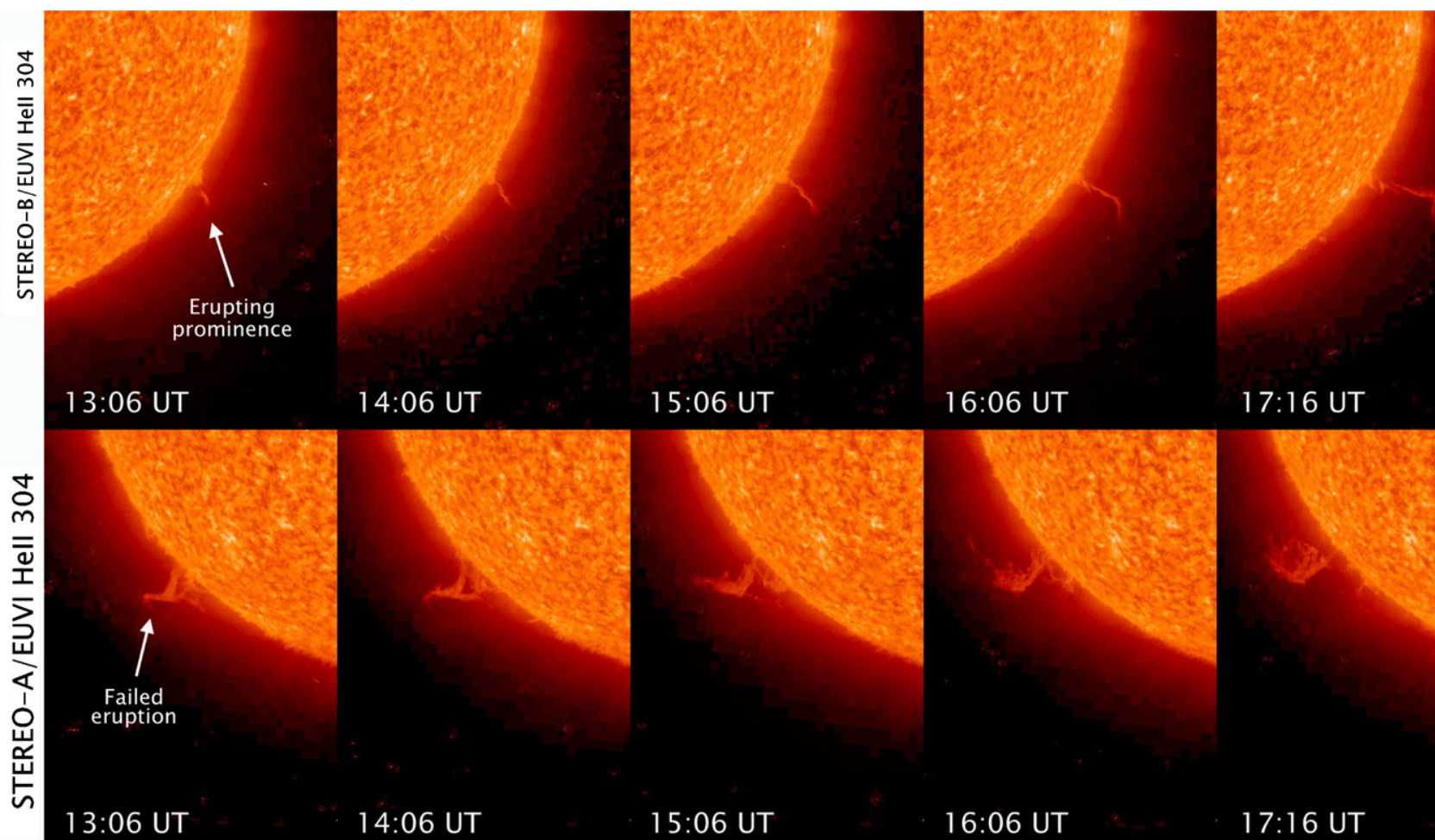

14:06 UT

15:06 UT

16:06 UT

17:16 UT

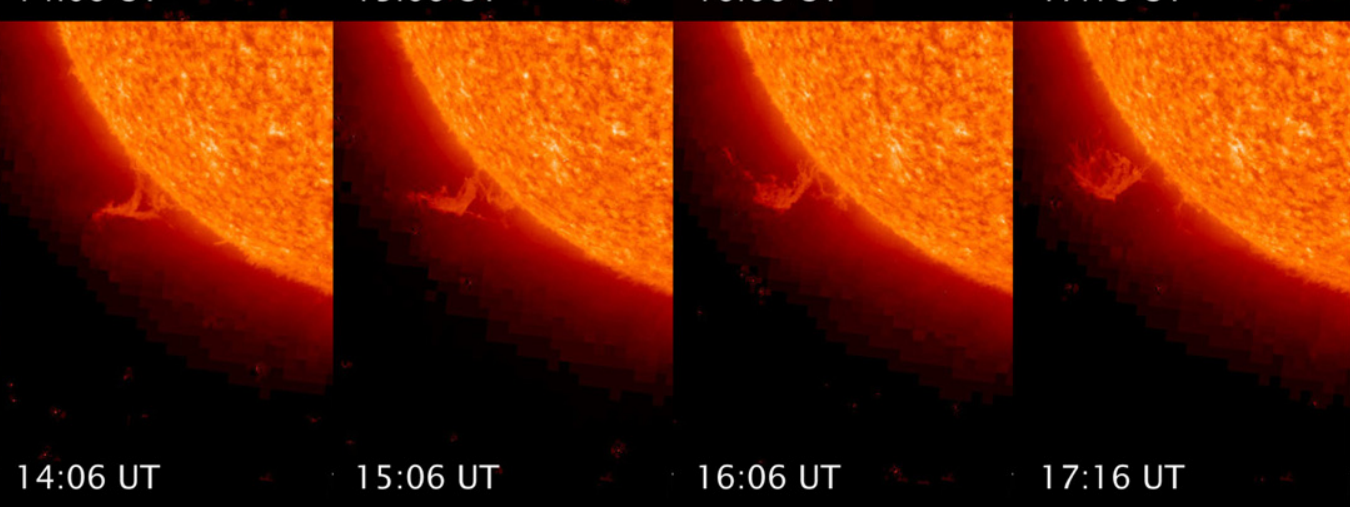

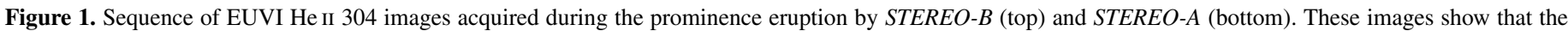

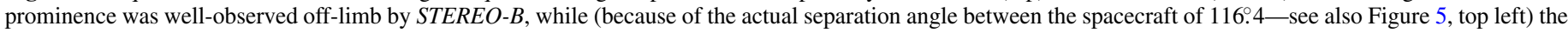
same material projects on-disk in the STEREO-A FOV (see the text and Figure 5).

(A color version of this figure is available in the online journal.)

rotation was followed by a faster expansion phase, and the erupting material eventually left the STEREO-B/EUVI FOV at a projected latitude of $\simeq 25^{\circ}$ south, around 19:37 UT.

At that time, the angle between the two STEREO spacecraft was approximately 116.4 , hence the material seen above the West limb by STEREO-B/EUVI, should have been seen by STEREO-A/EUVI on-disk approximately at a longitude of $26^{\circ}$ east. In fact, STEREO-A/EUVI He II 304 images do not show any erupting prominence above the limb (Figure 1, bottom panels), and the chromospheric material seen off-limb by STEREO-A is just a "failed eruption," i.e., plasma being ejected along some closed field lines and flowing back toward the Sun in the following hours. Hence, as we will better describe later, identification of the same erupting material in both STEREO-A and STEREO-B images will require a careful inspection of EUVI He II running difference images.

Because the prominence eruption is well observed above the west limb in STEREO-B/EUVI images, we expect the prominence to lie approximately on the STEREO-B plane of the sky (POS). On 2009 September 21, the STEREO-B-Sun-SOHO angle was $\simeq 55.6$ and as such the erupting prominence should have been observed by $\mathrm{SOHO}$ instruments on-disk approximately at a longitude of $\simeq 35^{\circ}$ west, in the south hemisphere. An inspection of $\mathrm{SOHO} /$ Michelson Doppler Imager (MDI) full disk magnetograms shows that at 14:24 UT a very small bipolar AR is located at $\sim 38^{\circ} \mathrm{S}, 15^{\circ} \mathrm{W}$; this AR is not registered in the NOAA catalogs. $\mathrm{SOHO} / \mathrm{EUV}$ Imaging Telescope (EIT) FexII 195 images (acquired with a temporal cadence of 12 minutes) show a very small system of loops connecting the two opposite magnetic polarities: the loops show intermittent brightenings before and during the eruption, but no clear activity (e.g., flares or EUV dimming) correlated with the eruption was observed in the EIT images. On 2009 September 21, only four frames were acquired by EIT with the He II 304 filter, and with this time cadence it was not possible to identify the erupting prominence material in these images. Nevertheless, as we will show later, the identification of this AR as the source region will be demonstrated by the $3 \mathrm{D}$ trajectories of the erupting prominence as derived by STEREO data analysis.

Higher up in altitude, a slowly evolving, balloon-type CME (Srivastava et al. 1999) was observed on SECCHI/COR1 images. The SECCHI/COR1 coronagraph is a classic Lyot internally occulted coronagraph which observes the white-light corona from 1.4 to $4 R_{\odot}$ (Thompson et al. 2003; Thompson $\&$ Reginald 2008). The coronagraph includes a linear polarizer which is used to extract the polarized brightness signal from the solar corona. The nominal spatial resolution is 7.5 arcsec (pixel size of $3.75 \mathrm{arcsec}$ ). The COR1 images, $2048 \times 2048$ pixels, are $2 \times 2$ binned on board before telemetering to the ground. The polarized brightness is extracted from three sequential images taken with polarizations of $0^{\circ}, 120^{\circ}$, and $240^{\circ}$. The cadence of a sequence is every 5 minutes. The CME was better observed in COR1-B images as a classical three-part structure event, with a bright leading edge, dark cavity, and a bright core (Figure 2, top panels). The CME entered the instrument FOV around 19:45 UT being observed (as a three-part structure) until 00:10 UT on September 22, while the erupting core was visible until $\sim 01: 35$ UT. The CME core first appeared above the COR1 occulter at a projected latitude of $\simeq 25^{\circ}$ south, in very good agreement with the prominence latitude leaving the EUVI-FOV at the same time. The core expanded northward until 22:30 UT, when the top of the core was at a projected latitude of $15^{\circ}$ south, hence closer to the equatorial plane. Interestingly, at later times the CME core was clearly deflected toward the equator, and 

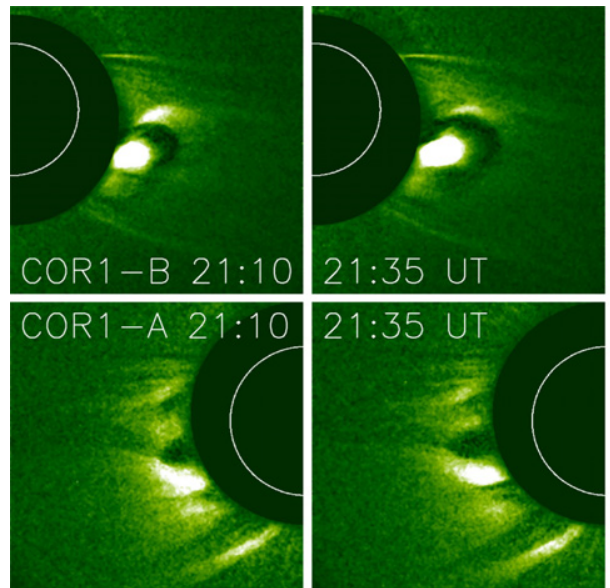
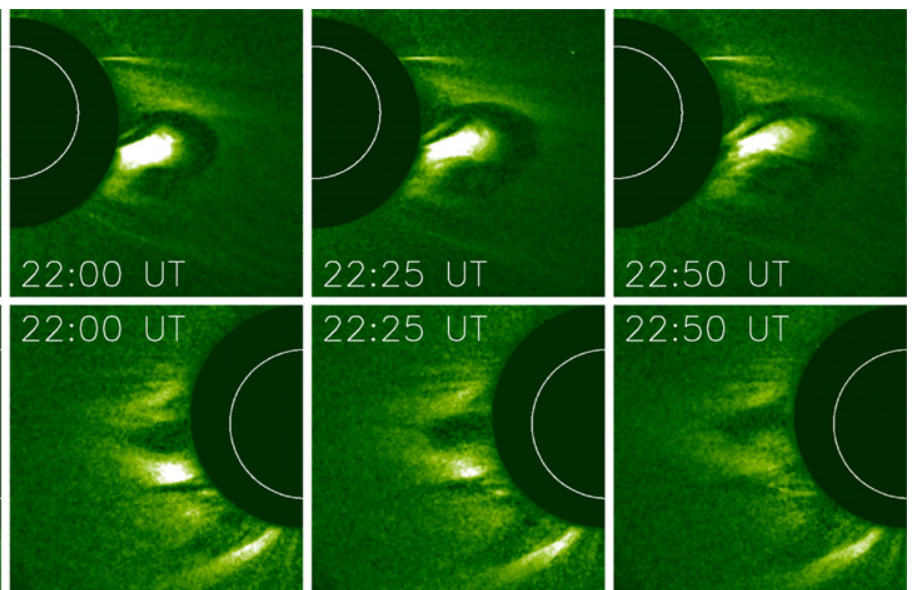

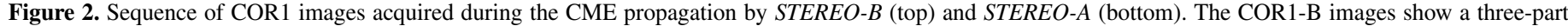

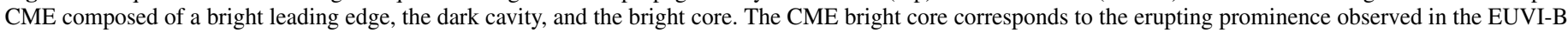

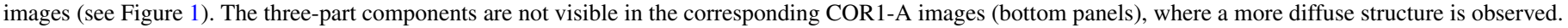

(A color version of this figure is available in the online journal.)

at around 23:00 UT it expanded outward almost radially. The CME was finally observed on 2009 September 22 by the COR2$\mathrm{B}$ instrument as a faint three-part structured bubble expanding along the equatorial plane.

The CME was much more diffuse in COR1-A images and the three-part components were not as clearly observed compared with COR1-B images (Figure 2, bottom panels). This is likely due to the large separation angle between the STEREO-A and STEREO-B spacecraft, making the CME, which expanded closer to the STEREO-B POS, very faint in the STEREO-A data. In COR1-A, a moving dark cavity started to be observed above the east limb around 20:00 UT at a projected latitude of $\simeq 24^{\circ}$ south. In the following minutes, the cavity slowly expanded outward in altitude and northward in latitude, moving toward the equator. This expansion mimics the motion of the CME core as observed by STEREO-B: the cavity first reached the equator, then at around 23:00 UT it expanded outward radially. Hence, STEREO/ COR1-A images confirm that the CME was deflected toward the equator while moving in the COR1 FOV.

A faint feature was also detected in the $\mathrm{SOHO} / \mathrm{LASCO} \mathrm{C} 2$ coronagraph. In particular, starting from $\sim 20: 30$ UT running difference images show a very faint arch-shaped feature, with an angular extension of $\sim 90^{\circ}$ expanding in the southwest quadrant. The good detection in STEREO/COR1-B images suggests that the CME was lying mainly on the STEREO-B POS, hence this faint feature detected by $\mathrm{SOHO} / \mathrm{LASCO} \mathrm{C} 2$ is likely the flank of the $\mathrm{CME}$ being closer to the SOHO POS. In the following frames, this faint feature mixes with a more intense second CME, observed on 2009 September 22, hence it was not possible to clearly identify the three-part components in LASCO images.

\subsection{Magnetic Field and Helicity Injection}

In order to investigate the magnetic evolution of the CME source region, we used full disk LOS $\mathrm{SOHO/MDI} \mathrm{magne-}$ tograms taken at $6767.8 \AA$ with a spatial resolution of 3.96 arcsec and a temporal resolution of 96 minutes. We considered magnetograms taken between 23:40 UT on 2009 September 18 and 22:37 UT on 2009 September 22. During this period, the region of interest spans over a longitude not bigger than $\pm 35^{\circ}$. For our analysis, we considered sub-fields with a FOV of $238 \times 238$ arc$\sec (120 \times 120$ pixels $)$ centered around the polarity inversion line of the small AR. We corrected our data for the inclination angle, i.e., the angle between the vertical magnetic field and the observer's LOS, and we aligned the sub-fields by applying a standard solar differential rotation rate (Howard et al. 1990) with a spatial resolution of 1 arcsec. Figure 3(a) shows the region of interest. The AR has an overall bipolar configuration having a positive leading polarity and a negative following polarity. Both polarities present a quite fragmented structure with a total negative flux slightly greater than the positive one (about $15 \%-20 \%)$. At the beginning of the observation, the negative polarity is more fragmented than the positive one. However, during the evolution, the negative polarity becomes more and more compact and in the meantime shows a southeast drift motion that results in a bigger separation between the two polarities. To derive the photospheric velocity maps, we used the differential affine velocity estimator (DAVE) with an FWHM of the apodization window of 19.8 arcsec (Schuck 2005). Figure 3(b) shows the derived velocity field for the magnetograms taken between 14:27 UT and 16:03 UT on 2009 September 20.

The helicity accumulation through the photosphere can be calculated as the time integral of the helicity density flux derived from a sequence of magnetograms. In particular, Pariat et al. (2005) presented a new definition of the helicity density flux that reduces the fake polarities in the helicity density flux maps. This helicity density is given by (Pariat et al. 2005)

$$
G_{\theta}(\mathbf{x})=-\frac{B_{n}}{2 \pi} \int_{S^{\prime}} \frac{d \Theta(\mathbf{r})}{d t} B_{n}^{\prime} d S^{\prime},
$$

where $\frac{d \Theta(\mathbf{r})}{d t}=r^{-2}\left(\mathbf{r} \times\left(\mathbf{u}-\mathbf{u}^{\prime}\right)\right)_{n}$ is the rotation rate of the two points located at $\mathbf{x}$ and $\mathbf{x}^{\prime}$, separated by a distance $r$, moving with velocities of $\mathbf{u}$ and $\mathbf{u}^{\prime}$ and having a normal component of the magnetic field of $B_{n}$ and $B_{n}^{\prime}$, respectively. Figure 3(c) shows such a helicity density map for the magnetograms taken between 14:27 UT and 16:03 UT on September 20. As is evident from the figure, the major helicity source corresponds to the negative polarity that is subjected to photospheric shearing motions. These shearing motions and helicity injection persist for quite a long time. The integral over the surface of interest of $G_{\theta}(\mathbf{x})$ is the helicity injection rate. The time integral of this quantity gives the helicity accumulation into the coronal volume. Figure 4 shows the helicity accumulation as a function of time for the 4 days of observations. After a first day during 


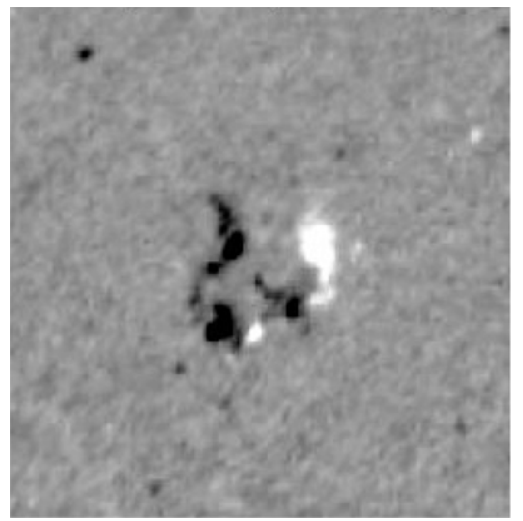

(a)

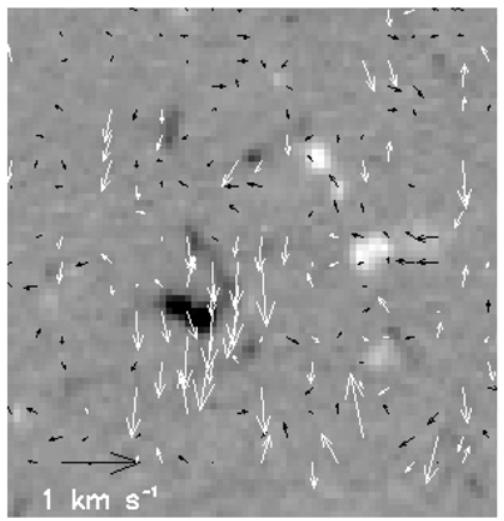

(b)

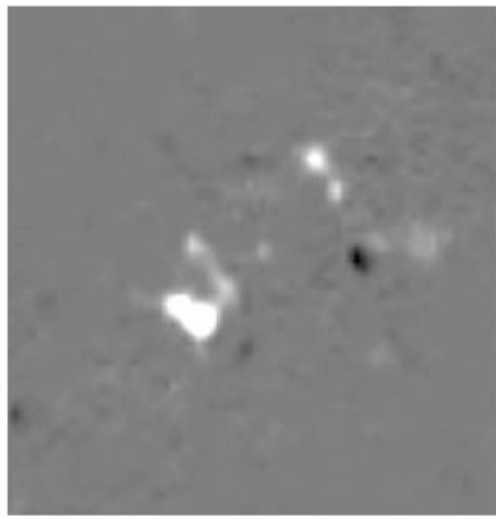

(c)

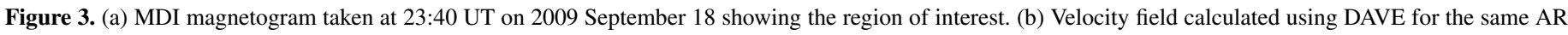

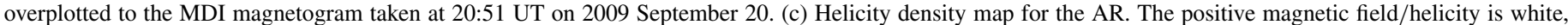
while the negative magnetic field/helicity is black. The field of view is $200 \times 200 \mathrm{arcsec}$.

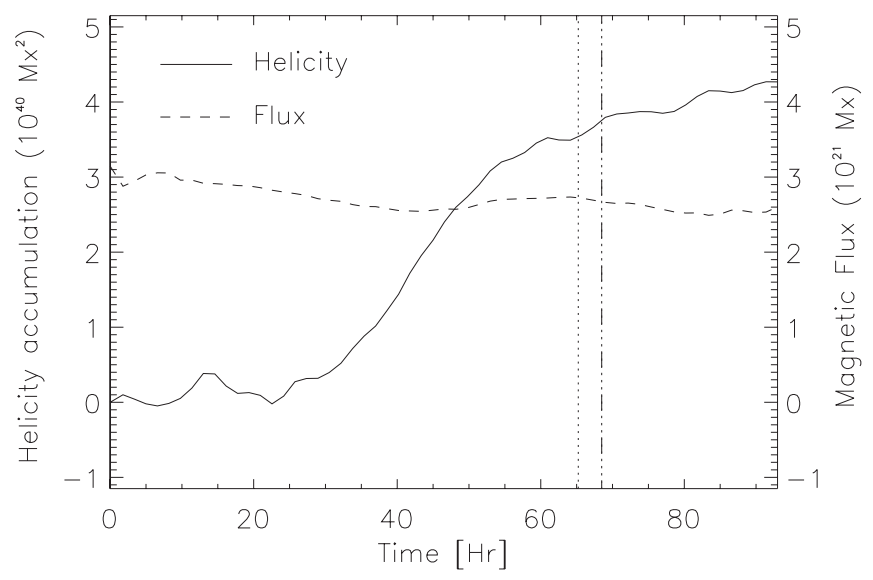

Figure 4. Helicity accumulation (solid line) and total magnetic flux (dashed line) as a function of time for the selected AR. The vertical dotted line indicates the moment at which the prominence leaves the EUVI-B field of view, while the vertical triple-dot-dashed line indicates the moment the CME's core reaches an altitude of $2.25 R_{\odot}$. Time zero is 23:40 UT on 2009 September 18 .

which no significant amount of helicity is injected through the photosphere, we observe a period of 1.5 days during which, almost linearly, an amount $\Delta H \simeq 4 \times 10^{40} \mathrm{Mx}^{2}$ of helicity is injected into the corona. This is in agreement with the helicity density flux map shown in Figure 3(c). At the end of this period of 1.5 days, i.e., on September 21 at 17:00 UT, the CME is launched (vertical dotted line). For the remaining 1.5 days after the eruption, we still observe positive helicity injection, but at a slower rate. During the same period, no significant flux emergence is observed as is illustrated in Figure 4, where the total magnetic flux for the selected area as a function of time is shown. The previous discussion brings us to the conclusion that, for this event, the major sources of helicity injection are the photospheric shearing motions to which the negative polarity is subjected. As a final remark, we want to stress that the value of the helicity injected into the corona is definitely smaller compared with bigger ARs (Smyrli et al. 2010). This further confirms the ephemeral nature of the region studied.

\subsection{D Reconstruction}

The 3D trajectory of the prominence and of the resulting CME has been reconstructed with $S T E R E O$ data. As mentioned above, the erupting prominence was observed off-limb by STEREO-B and on-disk by STEREO-A. Hence, in order to identify the prominence eruption on-disk in the STEREO-A images, we need to build $\mathrm{He}$ II 304 running difference images. A zoom over a couple of STEREO-A and STEREO-B running difference images showing the location of the erupting prominence in both instruments is shown in Figure 5. For triangulation, we used the standard scc_measure.pro routine provided with the STEREO package of the SolarSoftware library. This routine, after reading in a pair of STEREO EUVI-A and EUVI-B images, is able to trace the LOS of a point selected in one image pair into the FOV of the second image (the so-called epipolar line; see Inhester 2006 for details on the epipolar geometry). Because the eruption as seen from STEREO-A is quite faint, we applied this routine to the running difference images. The prominence has quite an elongated shape, hence it has not been difficult to identify the correspondence between STEREO-A and STEREO-B images, even though the prominence was observed on-disk by $S T E R E O-A$. In fact, different points along the prominence seen by STEREO-B correspond to LOS lines cutting across the faint radial feature seen by $S T E R E O-A$, thus providing a clear identification of pair points along the prominence.

The prominence was well observed by both EUVI-A and EUVI-B instruments between 15:16 and 16:56 UT; during this time interval 11 EUVI He II frames are available. For each EUVI frame, we selected typically 5-10 points along the prominence body (depending on the prominence contrast in EUVI-A with respect to the background), in order to reconstruct its $3 \mathrm{D}$ shape at different times. Results show that the prominence material leaves from a latitude of $34.4 \mathrm{~S}$ and a longitude of $20.9 \mathrm{~W}$ in the first frame we analyzed (15:16 UT). Hence, at that time the prominence material is located just a few degrees northward and westward with respect to the location of the AR we identified as the source of the eruption $\left(38^{\circ} \mathrm{S}, 15^{\circ} \mathrm{W}\right)$. In the following minutes, the prominence expands northward, moving mainly in a meridional plane (hence no significant longitudinal deflection is observed).

For the COR1 instrument, as it was difficult to identify the same features in A and B images and to apply the triangulation technique, we used the polarized ratio (PR) method (Moran \& Davila 2004; Dere et al. 2005) in order to derive the 3D structure of the CME. We applied the method on A and B images separately and we identified the top of the CME core (as observed in COR1-B images) for plotting the height-time (HT) diagram. The visible emission of the K-corona originates from the scattering of photospheric light by the free electrons in the 

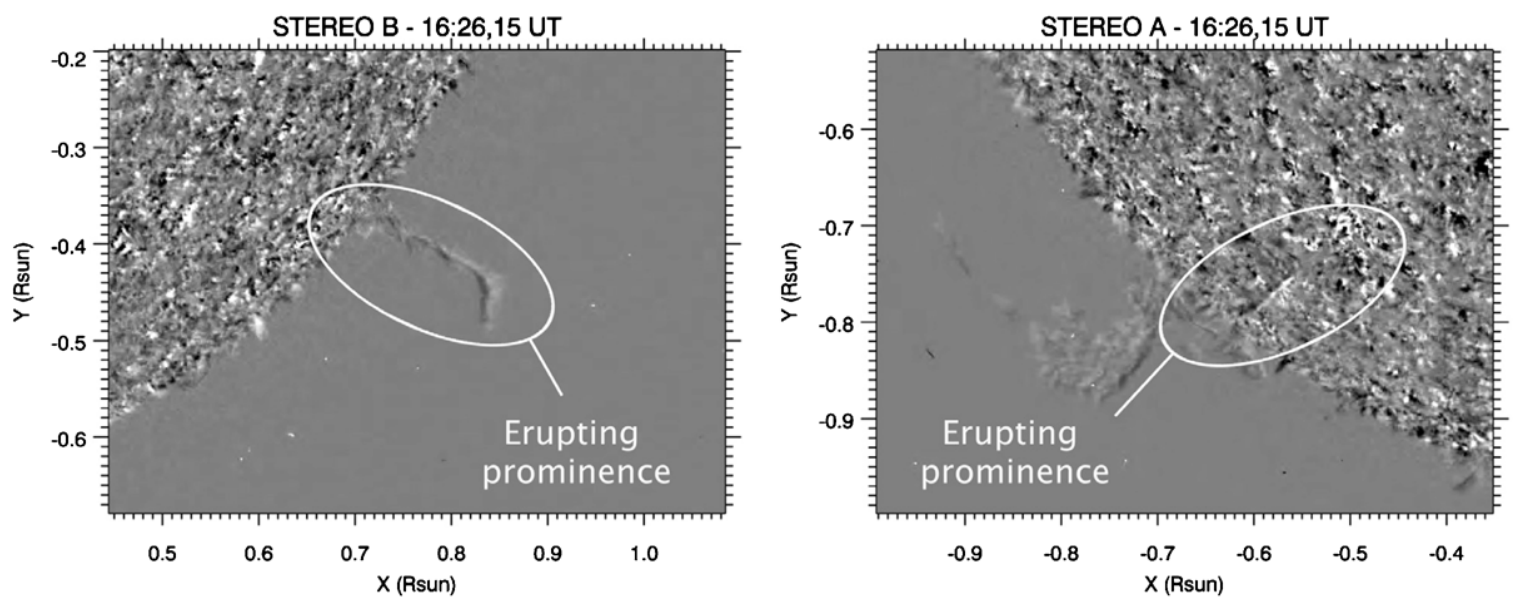

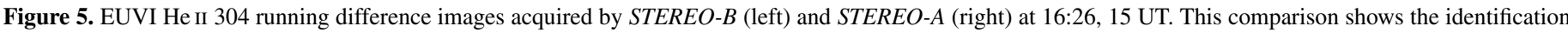

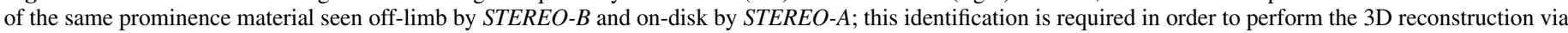
triangulation technique.

solar corona (Minnaert 1930; Van de Hulst 1950; Billings 1966) via the Thomson scattering mechanism. Recently, Mierla et al. (2011) reported that, in the case of the core of CMEs, a big part of this emission can be $\mathrm{H} \alpha$ emission. If prominence material is not present in the structure of a CME, then the emission should be due only to Thomson scattering. This is known to have a well-defined geometry (see, e.g., Billings 1966) and from this, the $3 \mathrm{D}$ structure of the CME can be inferred (Moran \& Davila 2004; Dere et al. 2005).

Each of the STEREO coronagraphs takes polarized images regularly and we can apply the method independently to the data from both spacecraft. The brightness ratio we use is the ratio of the polarized brightness $\mathrm{pB}$ to the unpolarized brightness $\mathrm{uB}$ ( $\mathrm{uB}=\mathrm{tB}-\mathrm{pB}$, where $\mathrm{tB}$ is the total brightness). In each case, we subtract a minimum intensity image from the CME images. In this way, we isolate the white-light emission from the CME only. In addition, a $5 \times 5$ median filter is applied to enhance the signal-to-noise ratio of the difference images. At each pixel of an image, the $\mathrm{pB} / \mathrm{uB}$ ratio is calculated and compared with the theoretical value for the Thomson scattering as a function of the scattering angle. For the LOS direction of the respective pixel, the scattering angle can then be transformed to an equivalent distance from the POS. Note that this reconstruction gives a weighted mean distance of the CME plasma density along each LOS. Due to the forward/backward symmetry of Thomson scattering, the brightness ratio does not indicate whether the scatterer is in front of or behind the POS. This ambiguity can be resolved to some extent if it is known whether the CME has been launched at the front side or the back side of the Sun (e.g., using the EUV observations of the CME source region).

Forward modeling (FM) was applied on the leading edge of the CME in order to resolve the forward/backward ambiguity of the PR method. The results that fitted both methods (FM and $\mathrm{PR}$ ) place the CME in front of the POS as observed by COR1-B. Then we take the average value of the points that constitute the top part of the CME core. Figure 6 shows the 3D distribution of points obtained with both triangulation and polarization ratio techniques, together with a fitting curve obtained with standard polynomial fitting (solid line). These points are shown in three views, as it was observed by STEREO-A and STEREO-B (bottom panels) and as was observed by $\mathrm{SOHO}$ (top right panel). The coordinate reference system in this figure is the standard Heliocentric System, having the $z$-axis pointing from the Sun toward the Earth, the $x$-axis pointing from the east to the west limb (as seen from Earth) and the $y$-axis pointing from solar south to north. The plot shows that the CME core propagates at a constant longitude (around $15^{\circ}$ west, in agreement with the prominence longitude), while a latitudinal deviation of about $15^{\circ}$ (from $-11^{\circ}$ at $2.2 R_{\odot}$ to $+3^{\circ}$ at $3.6 R_{\odot}$ ) is also observed.

\section{SIMULATIONS}

\subsection{Initial Condition}

In order to reproduce the key characteristics of the described event, the ideal MHD equations are solved numerically on a spherical, axisymmetric (2.5D) domain covering the region between the solar north and south poles, i.e., $(r, \vartheta) \in\left[1 R_{\odot}, 30 R_{\odot}\right] \times[0, \pi]$. The domain is discretized by a non-equidistant grid with $480 \times 201$ cells. A logarithmic grid stretching is used in both the radial and angular direction. A first to last grid cell ratio of $1 / 135$ is used in the radial direction, while for the co-latitude, the ratio for the equator to polar cell size is 0.3 . All simulations are performed with a modified version of the Versatile Advection Code (Tóth 1996), using a two-step Runge-Kutta scheme in time, while for the spatial discretization we use a second-order finite volume scheme, viz., the so-called total variation diminishing Lax-Friedrichs method. The magnetic field of the solution is maintained divergence free by exploiting an approach similar to that of Evans \& Hawley (1988). However, instead of storing the magnetic field components $B_{r}$ and $B_{\vartheta}$ on a staggered mesh, we use the vector potential $A_{\varphi}$ in the nodes. The same methodology was described in van der Holst et al. (2007).

The system of equations is solved in a frame corotating with the Sun, introducing centrifugal and Coriolis source terms in the equations, in addition to the gravitational source term. In order to reproduce a bimodal solar wind structure, an additional volumetric heating/cooling term resembling the effect of radiative losses, thermal conduction, and other heating mechanisms is added to the energy equation. The latter source term is similar to the one introduced in Groth et al. (2000) and Manchester et al. (2004), and was described in detail in several previous papers (e.g., van der Holst et al. 2007; Zuccarello et al. 2009).

In our simulation, the initial condition for the magnetic field consists of a dipole with a strength of $1.66 \mathrm{G}$ at the poles and with its magnetic dipole moment oriented anti-parallel with 

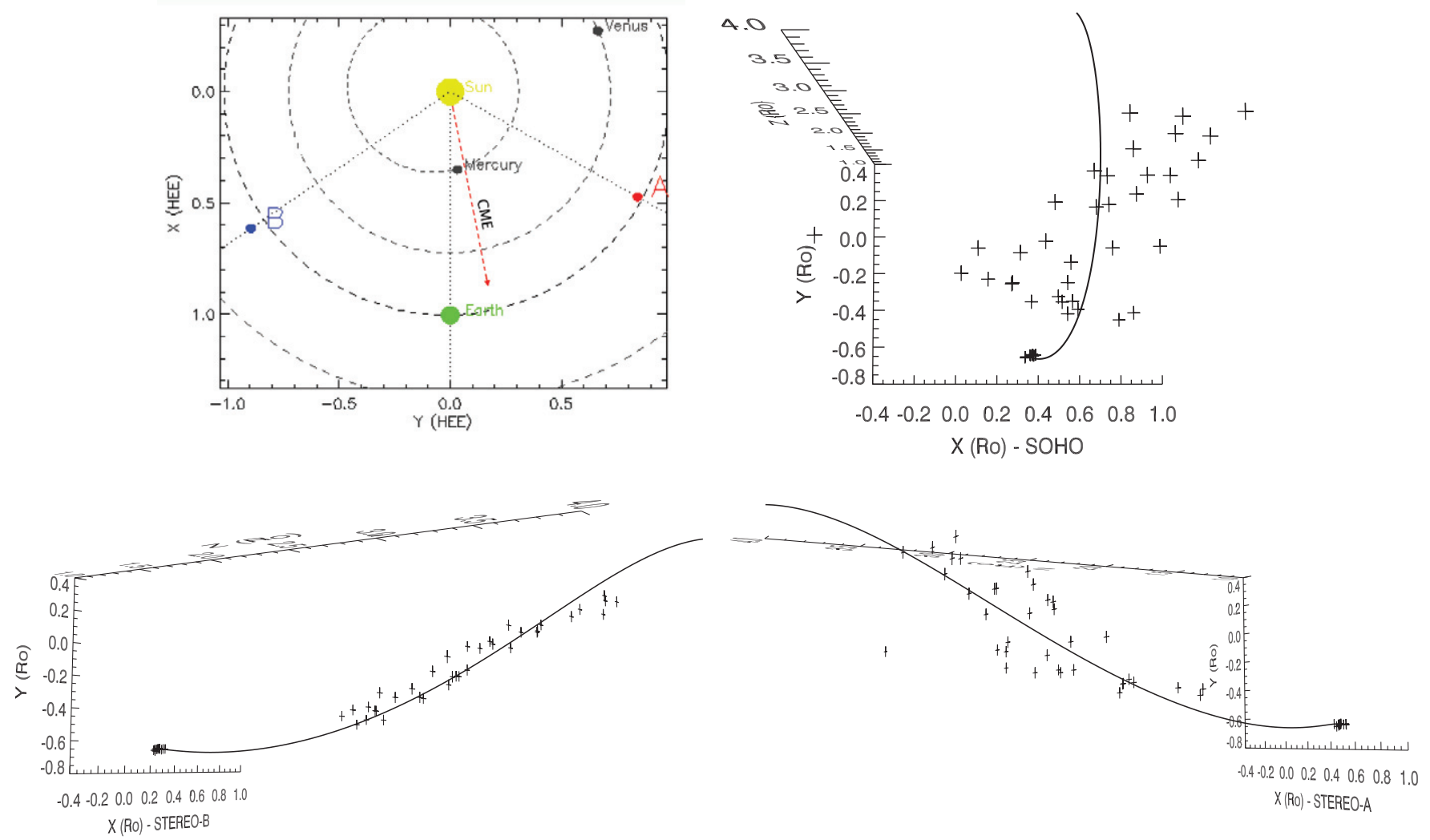

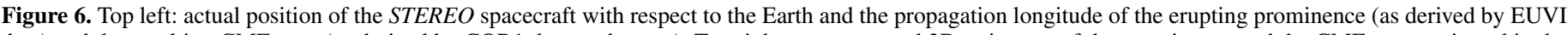

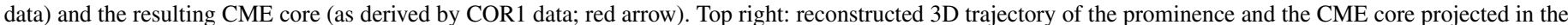

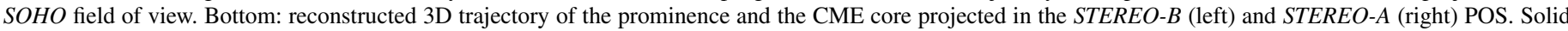

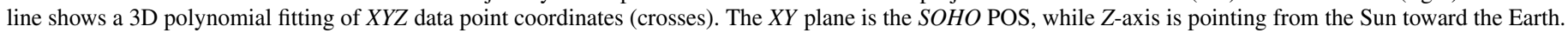
(A color version of this figure is available in the online journal.)

respect to the solar rotation axis. In the region with latitude $\lambda \in[-0.85,0.45] \mathrm{rad}$ an extra term is added to the vector potential of the dipole field, given by

$$
A_{\varphi}=\frac{A_{0}}{r^{4} \sin \vartheta} \cos ^{2}\left(\frac{\pi(\lambda+0.2)}{2 \Delta a}\right),
$$

where $A_{0}=0.648 \mathrm{G} \cdot R_{\odot}^{5}$ and $\Delta a=0.65 \mathrm{rad}$. The additional term in the vector potential creates a new bipolar flux system in the southern hemisphere, with a maximum magnetic field strength of about $1 \mathrm{G}$ on the solar surface.

At the inner boundary, both the density and the temperature are kept constant during the simulation. The density at the coronal base is $\rho_{0}=1.67 \times 10^{-16} \mathrm{~g} \mathrm{~cm}^{-3}$, while the temperature is $T_{0}=1.5 \times 10^{6} \mathrm{~K}$. The radial component of the momentum, if not negative, is extrapolated, otherwise it is fixed to zero in the ghost cells, while the poloidal component is always extrapolated. Since the frame is corotating with the Sun, the azimuthal component of the momentum is fixed to zero in the ghost cells. Finally, the vector potential is kept fixed at its initial value during the complete simulation. At the outer boundary, the flow is superfast, therefore open boundary conditions are applied to all the variables. The system is then advanced in time until a steady state is obtained, which is displayed in Figure 7(a).

The stationary solution consists of a helmet streamer that is slightly shifted northward with respect to the solar equator. The loop system associated with the AR is located south of the helmet streamer, and is separated from it by a region of open magnetic field with positive polarity. The negative polarity of the aforementioned AR is connected to the positive part of the global dipole by a smaller loop system compared to the northern arcade. Because of the southern shift of the central arcade, the current simulation setup significantly differs from the one presented in Zuccarello et al. (2009), which was symmetric with respect to the equator and contained an $X$-type null point, favorable for breakout reconnection. The weaker global field in combination with the southward shifted central arcade results in a large northern helmet streamer and two smaller arcade systems in the southern hemisphere that are embedded in a "pseudostreamer" structure (Wang et al. 2007). Contrary to the helmet streamer which stretches out in a thin current sheet over one neutral line, the pseudostreamer extends over two neutral lines, separating coronal holes of the same polarity.

As a proxy for the observed coronal magnetic field structure, a PFSS extrapolation was used. The harmonic coefficients for the potential field were obtained via the pfss SolarSoftWare package (Schrijver \& DeRosa 2003). In Figure 7(b), the reconstructed field for 2009 September 19 at 12:04 UT is shown, where we have limited the order of the spherical harmonics used in the PFSS reconstruction to $l=10, m=10$. The figure shows the magnetic field lines and the strength of the radial magnetic field in the plane containing the center of the AR of interest. The reconstructed potential magnetic field presents a morphology similar to the initial magnetic configuration of the simulation. We would like to note that the key properties of the reconstructed field, i.e., the asymmetry between the two outer arcades, the northward shift of the cusp of the helmet streamer, and the southern pseudostreamer, are all reproduced. However, 


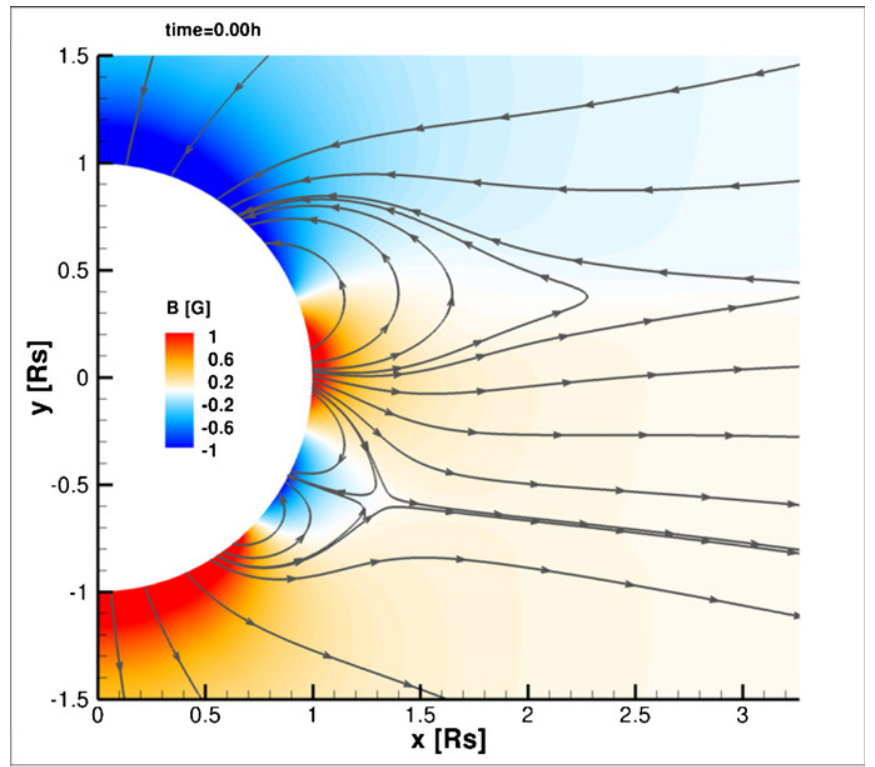

(a)

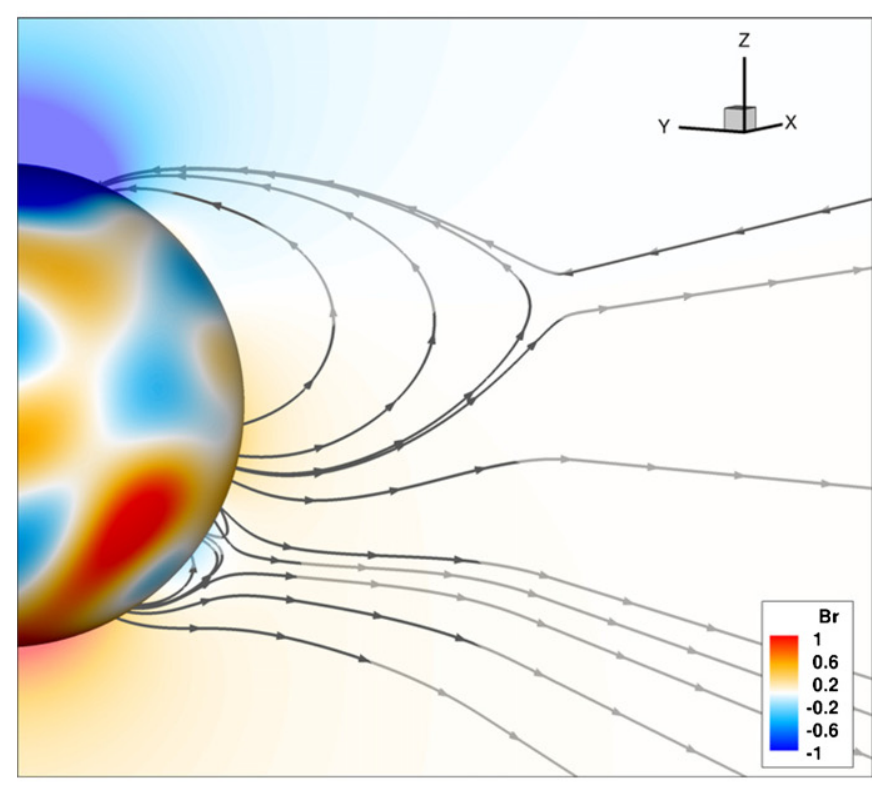

(b)

Figure 7. Magnetic field distribution (color scale) and selected field lines for (a) the steady state of the simulation and (b) the PFSS extrapolation obtained from the MDI data on 2009 September 19 at 12:04 UT.

(A color version of this figure is available in the online journal.)

some differences are present as well. First, the extrapolated magnetic field has an azimuthal component that is not present in our simulation because of the dimensional limitation to 2.5D. Second, the cusp of the helmet streamer presents an off-equator angle that is bigger than the one in the simulation.

\subsection{Boundary Driven Motions}

In Section 2.2, we investigated the magnetic evolution of the AR that was the source of the CME. We argued that shearing motions, mainly concentrated around the negative polarity, were the source of the magnetic helicity injection, and eventually the driving mechanism for this eruption. In fact, the PFSS extrapolation shows a magnetic arcade connecting the negative polarity of the AR with the southern large-scale field. These shearing motions in the negative polarity of the AR will increase the magnetic stress in the southern arcade. Therefore, we have applied localized shearing motions along the azimuthal direction in the southern loop system. During the driving phase, the vector potential is unmodified at the inner boundary (and as such the radial component of the photospheric magnetic field), representing the observed constant magnetic flux (see Figure 4).

At the inner boundary, in a region of $2 \Delta \vartheta=0.3 \mathrm{rad}$, centered around a latitude of $\vartheta_{0}=-0.7 \mathrm{rad}$, an azimuthal flow is imposed with a profile of the form

$$
v_{\varphi}=v_{0}\left(\alpha^{2}-\Delta \vartheta^{2}\right)^{2} \sin \alpha \sin \left[\pi\left(t-t_{0}\right) / \Delta t\right],
$$

where $\alpha=\frac{\pi}{2}-\vartheta_{0}-\vartheta$. The value of $\vartheta_{0}=-0.7$ rad corresponds to the latitude of the polarity inversion line of the southern arcade. The maximum shear velocity depends on the scaling factor $v_{0}$ and for the simulation the maximum shear flow, $v_{\max }$, was set to $16.75 \mathrm{~km} \mathrm{~s}^{-1}$. The shearing motions are gradually switched on at time $t_{0}=0$, reach a maximum at time $t=\Delta t / 2$, and are completely turned off after the time $\Delta t$. The time interval over which the shearing motions are applied is set to $\Delta t=36 \mathrm{hr}$. In fact, this is the time over which the more significant fraction of positive magnetic helicity is injected into the solar corona as shown in Figure 4.

As a final remark, we would like to stress that $v_{\max }$ is the value of the shear velocity at time $\Delta t / 2$ and at a latitude of $-40^{\circ} \pm 4^{\circ}$, so the average shear velocity is much less than this value. This value is still higher than what is observed at the photosphere, where the average shearing velocities are about $0.4-0.8 \mathrm{~km} \mathrm{~s}^{-1}$ (Figure 3(b)). However, the inner boundary of our simulation corresponds to the lower corona and not to the photosphere. This choice for the shearing velocity is a compromise between observational constraints and computational requirements that still satisfies the sub-Alfénic nature of the photospheric shearing motions.

\subsection{Dynamical Evolution}

After the steady state is reached, we impose the timedependent boundary condition discussed in the previous section. Figures 8 and 9 show the evolution of the azimuthal component of the current density and of the relative density at different moments during the evolution of the system. Even though we performed ideal MHD simulations, numerical diffusion is present in the code and will introduce reconnection.

Figure 8(a) shows the magnetic configuration of the system after $21.84 \mathrm{hr}$. The gray scale is the azimuthal component of the current density, while the different colors of the field lines indicate different flux systems. Regions of high current density indicate the reconnection location. As a consequence of the applied shearing motions, the magnetic pressure increases and the southern side arcade starts to expand. During this expansion, the null point in the pseudostreamer is pushed northward and one elongated current sheet is formed between the expanding southern arcade and the open field region at the north of it, eventually initiating the reconnection. As a consequence of this reconnection, the magnetic flux of the expanding southern arcade (orange field lines) is transferred partially to the central arcade (red field lines), which becomes bigger, and partially to the open flux of the northern helmet streamer (blue field lines). The result of this process of interchange reconnection is visualized in the figure by the cyan field lines, i.e., originally the closed field lines belonging to the southern arcade that now 


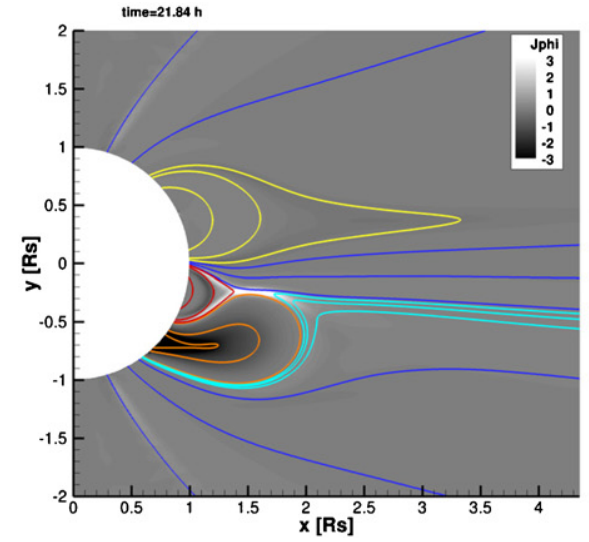

(a)

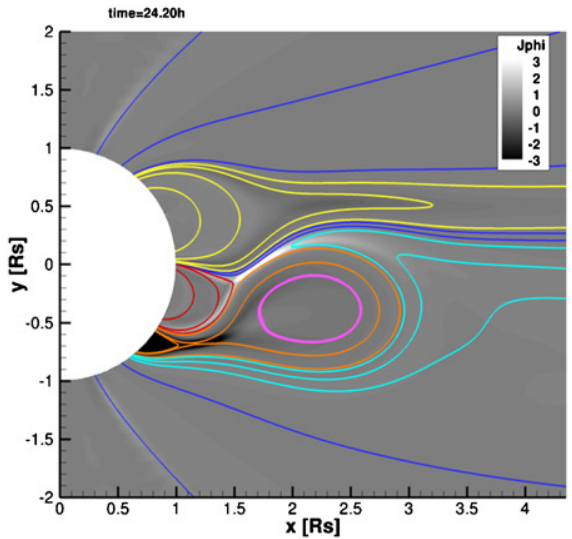

(b)

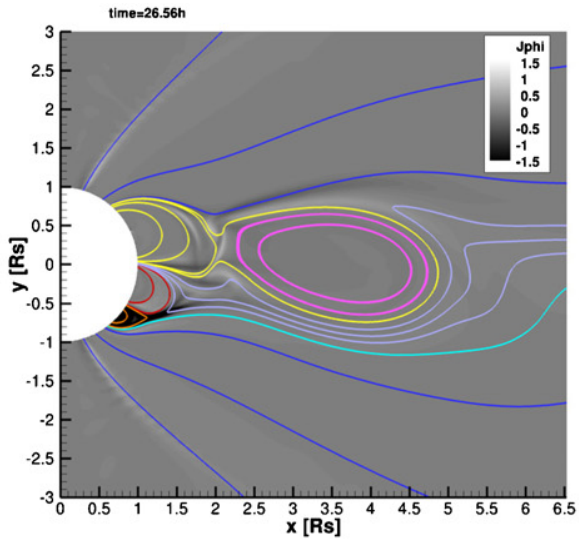

(c)

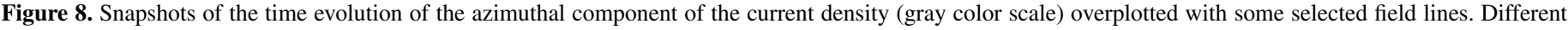
flux systems are identified by different colors (see the text for more details).

(A color version of this figure is available in the online journal.)

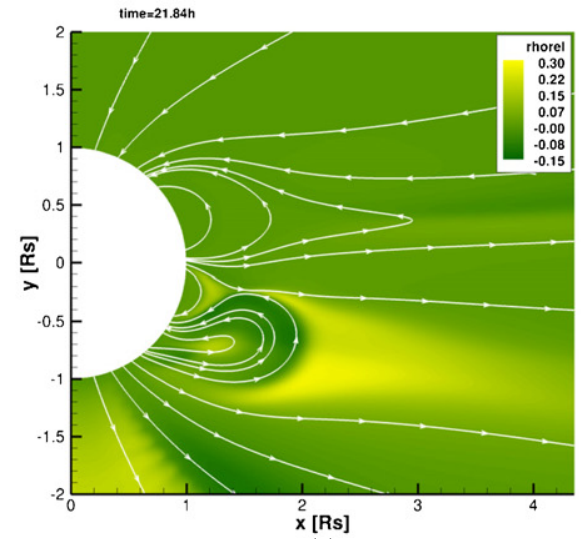

(a)

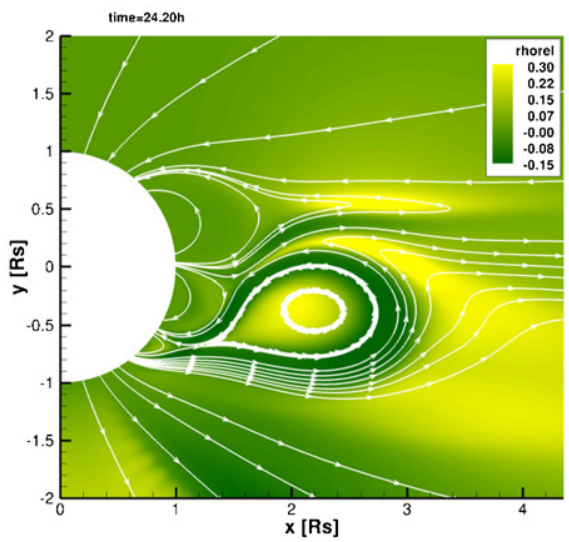

(b)

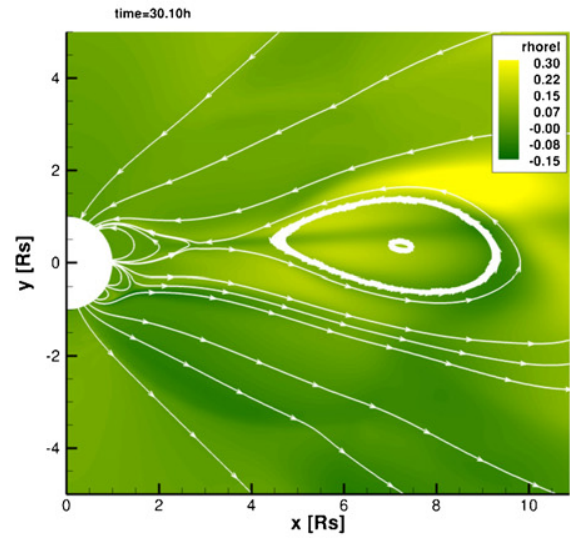

(c)

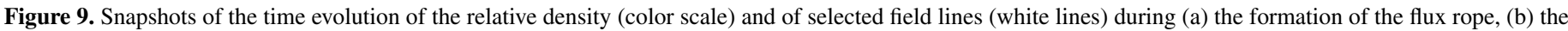
deflection toward the equator, and (c) during its propagation.

(A color version of this figure is available in the online journal.)

belong to the southern coronal hole. Figure 8(a) also shows how the flanks of the southern arcade (orange field lines) pinch together. In fact, the shear velocity is a function of the latitude, so that field lines with footpoints at different latitudes will be sheared at different velocities. This results in a nonuniform stressing of the field lines. The imbalance between the magnetic tension and the magnetic pressure forces causes the flanks of the inner field lines to be pinched together, ultimately resulting in the formation of a flux rope. A significant increase in the azimuthal component of the current density is observed in the center of the southern arcade, eventually increasing the magnetic free energy available for the eruption.

Figure 8(b) shows the current density distribution at $24.2 \mathrm{hr}$. The pinching at the flanks of the southern arcade resulted in the formation of the flux rope (pink field lines) and during this reconnection process more and more magnetic flux is transferred from the southern arcade to the flux rope. The reconnection at the upper part of the expanding arcade results in a magnetic pressure imbalance between the north and the south part of the side arcade that, as a consequence, is deflected toward the equator. At this stage, two main reconnection layers are present. One transfers flux from the side arcade to the flux rope (orange/pink field lines) and the other transfers flux of the expanded southern arcade partially to the central arcade (red/ orange/blue field lines) and partially to the southern coronal hole (cyan/orange/blue field lines). While these reconnections proceed, the central arcade continues to expand, because more and more flux is transferred to it. At a certain moment, due to the ongoing reconnection inside the southern arcade and the continuous growth of the central arcade, the newly formed open flux of the southern coronal hole (cyan field lines) will reconnect with the flux of the central arcade definitely separating the flux rope from its formation location. The magnetic configuration resulting from the interaction between the central arcade and the southern coronal hole is indicated in Figure 8(c) by the lilac field lines. Figure 8(c) also shows how the flux rope (pink field lines) gets absorbed in the northern helmet streamer when no more open field (blue field lines) is present between the flux rope field (pink field lines) and the helmet streamer (yellow field lines). After that, the CME propagates through the current sheet of the northern helmet streamer. While the CME propagates along the current sheet, it stretches the cusp of the northern arcade eventually transferring even more flux to the CME. The propagation occurs inside the overlying (yellow) field lines that are carried away together with the propagating flux rope.

Figure 9 shows the evolution of the relative density at different moments during the evolution of the system. As a consequence of the expansion of the southern arcade, an increase in the relative density is observed at the leading edge of the expanding loop system, while a density depletion is observed 


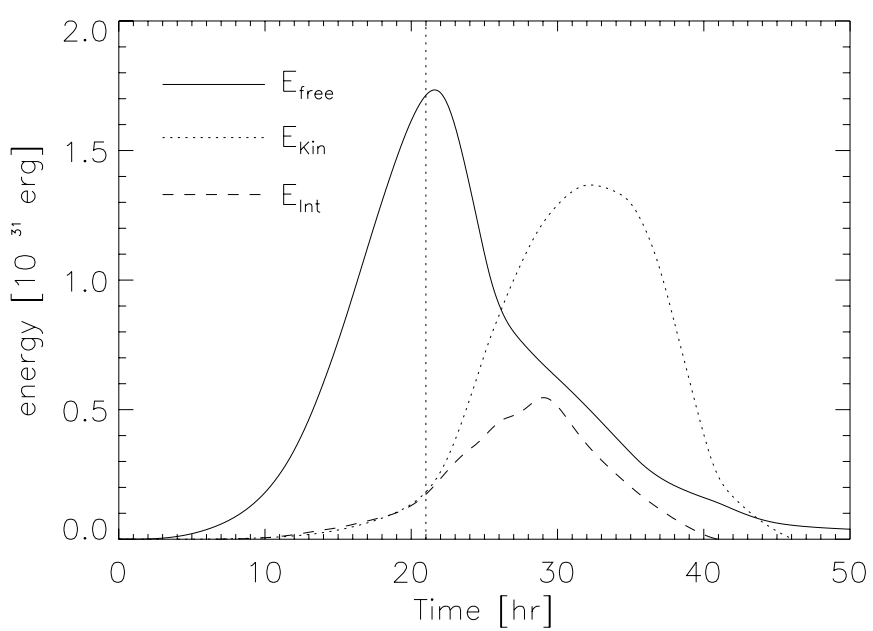

Figure 10. Time evolution of the magnetic free energy (solid line), the kinetic energy (dotted line), and the internal energy (dashed line) with respect to the energy in the steady state. The vertical dotted line indicates the moment in which the flux rope is accelerated.

behind it, as shown in Figure 9(a). The same figure also shows an increase in the relative density in the central arcade due to reconnection corresponding to the loop brightening observed in EUV images. Figure 9(b) shows the relative density configuration at $24.20 \mathrm{hr}$ when the flux rope is propagating within the COR1 FOV. The high-density core as well as the three-part structure are clearly visible. Moreover, during the propagation, as a consequence of the magnetic pressure imbalance described before, a clear deflection of the plasma embedded inside the flux rope is observed. This deflection causes the CME to approach the IP current sheet within the first $4 R_{\odot}$. After that, the $\mathrm{CME}$ propagates through the numerical domain as shown if Figure 9(c).

CMEs are magnetically driven phenomena. It is well accepted that the energy available for the eruption is the magnetic free energy. The magnetic free energy is the difference between the magnetic energy of a given magnetic field and the magnetic energy of the potential field having the same magnetic field distribution at the boundary, i.e., the energy stored in the currents. Figure 10 shows the evolution of the magnetic free energy, the kinetic energy, and the internal energy for the system. As a consequence of the applied photospheric shearing motions, the free magnetic energy rises. This is in agreement with the increase in the current density observed in the southern side arcade. After about $20 \mathrm{hr}$, a peak in the magnetic energy followed by a decrease is observed. This indicates the moment when the flare reconnection sets in and the flux rope is formed (dotted vertical line).

The dotted line in the figure shows the evolution of the kinetic energy with respect to the kinetic energy of the background solar wind. During the first $20 \mathrm{hr}$ of the driving phase, the kinetic energy of the system shows a modest increase mainly due to the plasma motions associated with the rise of the loop system of the southern arcade. However, when the reconnection sets in, magnetic energy is converted into kinetic energy that rises quite drastically. When the leading edge of the flux rope reaches the outer boundary, after about $11 \mathrm{hr}$ from the flux rope formation, the kinetic energy decreases, eventually approaching the initial steady state value again. Figure 10 also shows the evolution of the internal energy (dashed line). Also the internal energy of the system increases when the increase in the magnetic pressure of the southern arcade results in the formation of the leading edge of the CME. When the reconnection sets in, the internal energy increases even further until the moment at which the front of the flux rope reaches the outer boundary of the computational domain. Finally, the energy evolution of the system clearly shows that all the magnetic free energy built up as a consequence of the shearing motions was converted into both kinetic and internal energy and that the system relaxes back to its initial configuration after the CME has propagated out of the computational domain.

\subsection{Radial and Latitudinal Evolution}

During its initial evolution, the CME undergoes a deflection toward the equator. To follow both the latitudinal and the radial evolution of the flux rope during its expansion, we identified the center of the flux rope and followed its position in the meridional plane as a function of time.

Figure 11(a) shows the latitude of the center of the flux rope as a function of the height from the solar surface. The flux rope is formed at a height of about $1.35 R_{\odot}$ and at a latitude of about $-33^{\circ}$. Within $\simeq 3.5 R_{\odot}$ it is deflected toward the equator, eventually crossing it and reaching a latitude of about $+3^{\circ}$, interacting with the cusp of the northern helmet streamer. Afterward, the CME propagates through the current sheet, which is slightly inclined north, having a latitude of about $+5^{\circ}$.

Figure 11(b) shows the HT plot for the simulated CME. The flux rope is formed about $18 \mathrm{hr}$ after the start of the helicity injection, and within $3 \mathrm{hr}$ it moves from $1.35 R_{\odot}$ to a height of $2.25 R_{\odot}$. Next, it gets swept up by the solar wind and reaches the outer boundary at $30 R_{\odot}$ after about $35 \mathrm{hr}$. Figure $11(\mathrm{c})$ shows the velocity of the CME as a function of the altitude. The obtained CME is of the slow type and has a propagation speed of about $400 \mathrm{~km} \mathrm{~s}^{-1}$. The main acceleration phase occurs within the FOV of COR2, i.e., $15 R_{\odot}$, after that it propagates through the computational domain with an almost constant speed.

In Zuccarello et al. (2009), considering a breakout configuration symmetric with respect to the solar equator, we have shown that, as a consequence of the shearing motions applied along the polarity inversion line of the central arcade, a CME was initiated. We noticed that the obtained slow CME was the detached helmet streamer top that was carried away by the slow solar wind. In this configuration, the actual CME is formed from the side arcade and is actually accelerated, at least during the initial phase, by the flare reconnection. However, the obtained CME is slow. In the previous section, we have noticed that the newly formed flux rope, while approaching the solar equator, interacts with the northern helmet streamer eventually becoming part of it. Therefore, while propagating outward, the flux rope is slowed down by the extra magnetic tension of the magnetic field of the northern helmet streamer, eventually resulting in a slow CME.

\section{DISCUSSION}

The magnetic helicity, and in particular the relative magnetic helicity (Berger \& Field 1984), is a key quantity in the evolution of the magnetic field. In fact, it is a measure of the linkage and of the stress of a given magnetic field. In an axisymmetric domain, the relative magnetic helicity in the coronal volume is given by

$$
H=2 \int_{\mathcal{V}} A_{\varphi} B_{\varphi} d \mathcal{V}
$$

Figure 12 shows the evolution of the magnetic helicity in the system as a function of time. For the purpose of comparison 


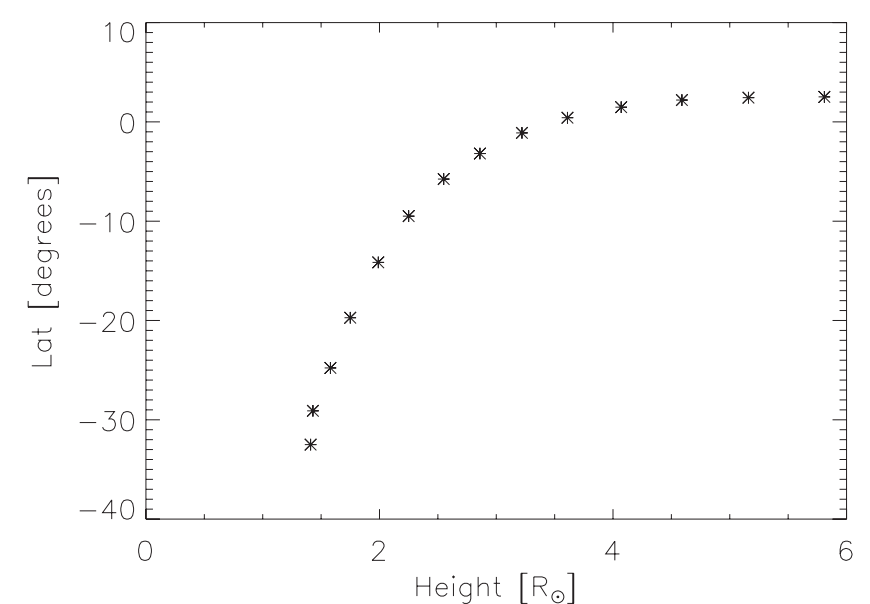

(a)

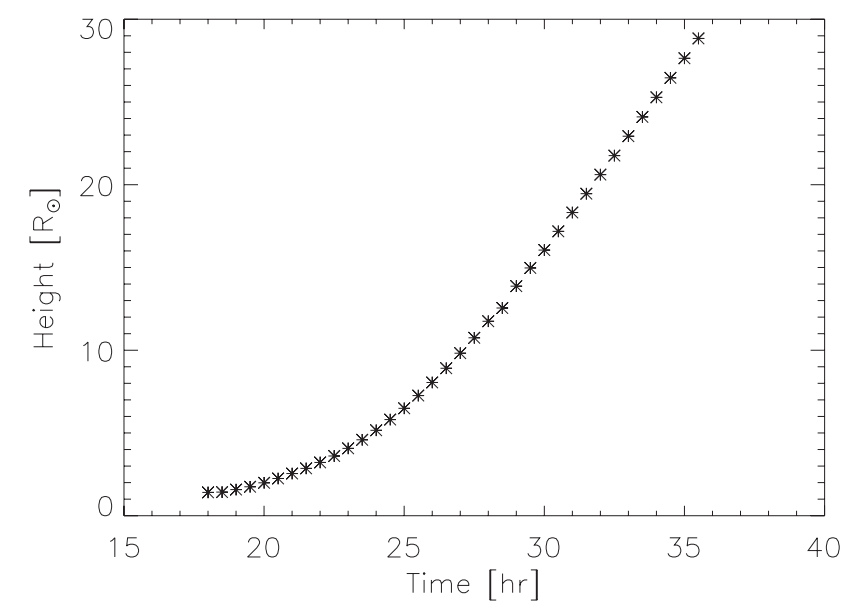

(b)

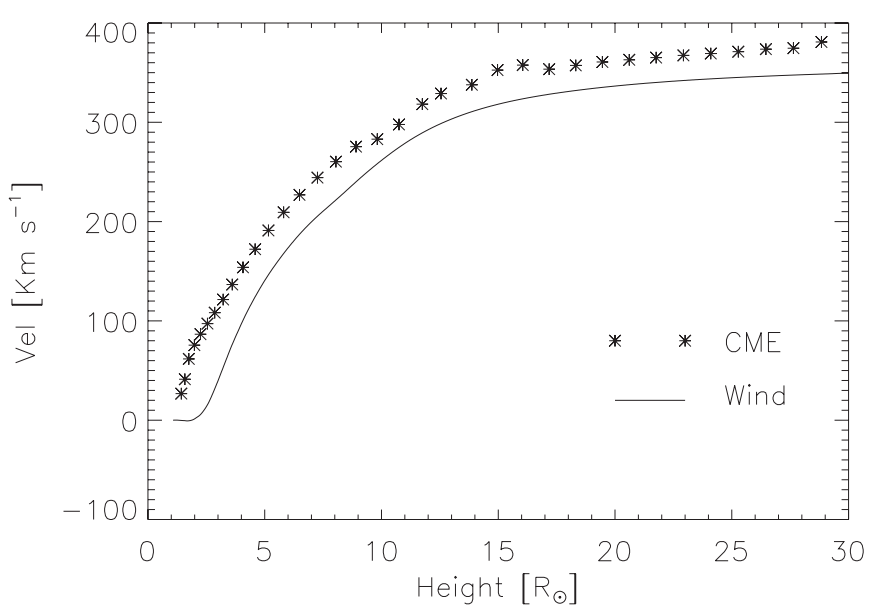

(c)

Figure 11. Evolution of the center of the flux rope. (a) Latitude vs. altitude, (b) altitude vs. time, and (c) velocity vs. altitude. For comparison, the background solar wind speed is also shown in frame (c).

with the observations the magnetic helicity is expressed in its natural units, i.e., the magnetic helicity is normalized with the square of the total unsigned magnetic flux at the solar base. We set the origin of the time axis at the moment at which the core of the CME has an altitude of $2.25 R_{\odot}$ in both the simulation and the observation. Positive magnetic helicity is injected into the system due to the shearing motions on a timescale of about $36 \mathrm{hr}$. After about $20 \mathrm{hr}$ enough stress has been introduced

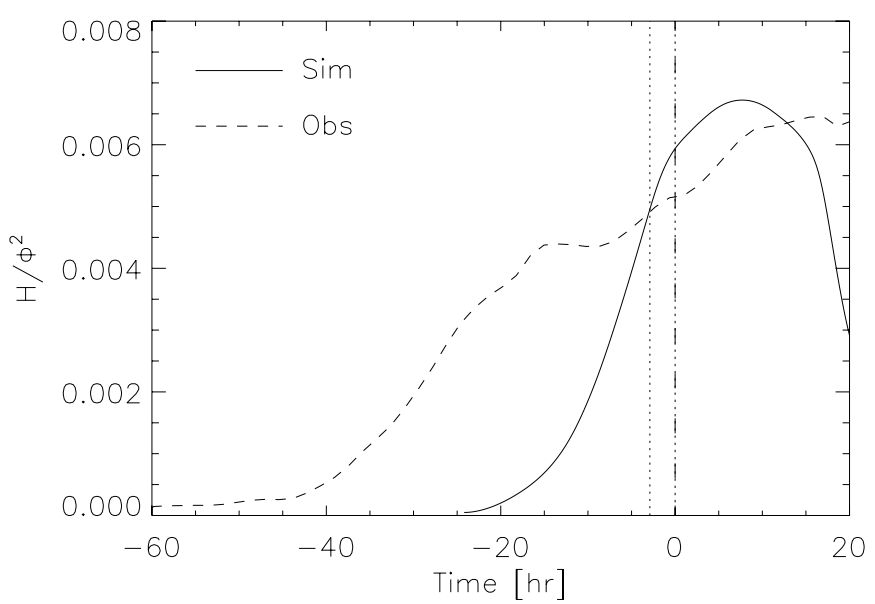

Figure 12. Time evolution of the normalized coronal helicity for both the simulation (solid line) and the observed event (dashed line). Time zero corresponds to the moment at which the CME was at $2.25 R_{\odot}$. The vertical dotted line indicates the moment in which the prominence leaves the EUVIFOV.

into the system and the flux rope is formed (vertical dotted line). However, shearing motions are still applied at the lower boundary and therefore helicity is still injected into the system. At a time of $6 \mathrm{hr}$, i.e., about $30 \mathrm{hr}$ after the shearing motions began, the magnetic helicity reaches a maximum and then starts to decrease. From $t=12 \mathrm{hr}$ on, no additional helicity is injected into the corona. A few hours after the shearing motions are stopped, the CME reaches the outer boundary and consequently the magnetic helicity significantly drops and will finally go back to zero (not shown in the figure). All the magnetic helicity injected as a consequence of the shearing motions has been transferred into the IP medium through the CME, eventually avoiding the endless accumulation of magnetic helicity in the solar corona.

Figure 12 also shows the helicity injection calculated in Section 2.2 normalized to the magnetic flux of the AR. The vertical triple-dot-dashed line indicates the moment at which the CME appears in the COR1 FOV. Due to the projection effects, this corresponds to an altitude of $2.25 R_{\odot}$. However, it also indicates the moment in which the center of the simulated flux rope reaches a distance of $2.25 R_{\odot}$. The vertical dotted line indicates the moment in which the filament was not visible anymore in the EUVI field of view. It is interesting to note that this moment coincides with the moment in which the simulated flux rope is formed and quickly accelerates. Moreover, when the simulated flux rope lifts off, the amount of helicity injected into the system is comparable with the amount of helicity injected into the solar corona when the filament disappeared from the EUVI-FOV.

Figure 13 shows the COR 1 image at time 22:15 UT (a) and the snapshot of the relative density at time $24.2 \mathrm{hr}$ for the simulation (b). Both the three-part structure and the deflection of the CME are well reproduced in the simulation. However, the simulation shows a high-density region at the interface between open and closed magnetic field lines. This high-density region, which is not present in the observations, is the result of the symmetry in the model. In fact, due to the axial symmetry, the flux rope is a torus around the Sun.

In order to compare the early stages of the dynamics of the event, Figure 14(a) shows the HT plot for both the simulation (dashed line) and the reconstructed CME ("plus" signs). The simulated flux rope has an HT evolution that is comparable 


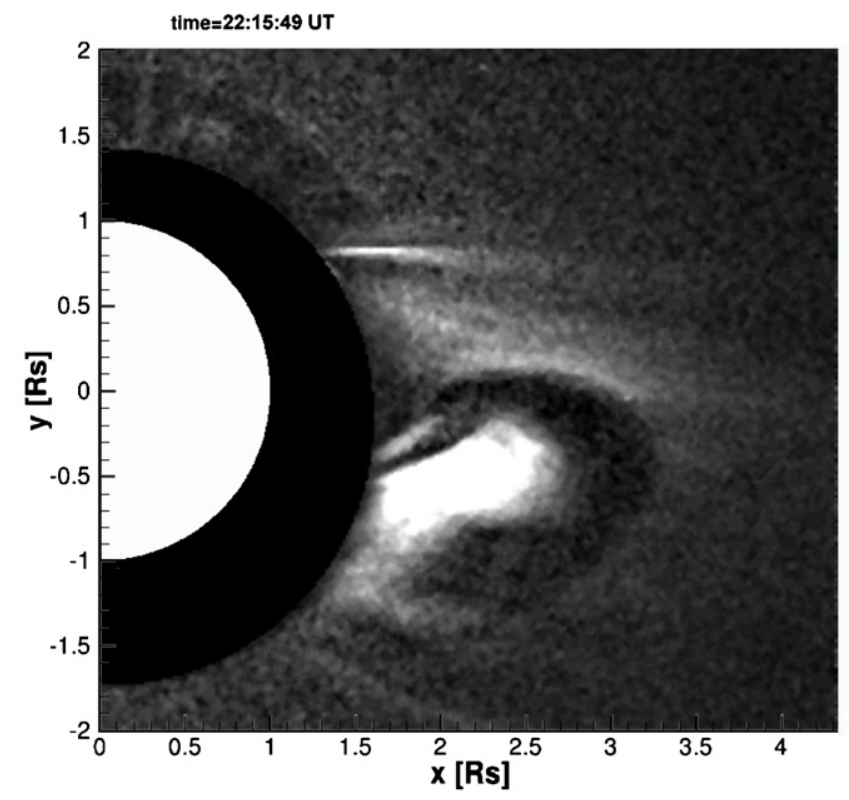

(a)

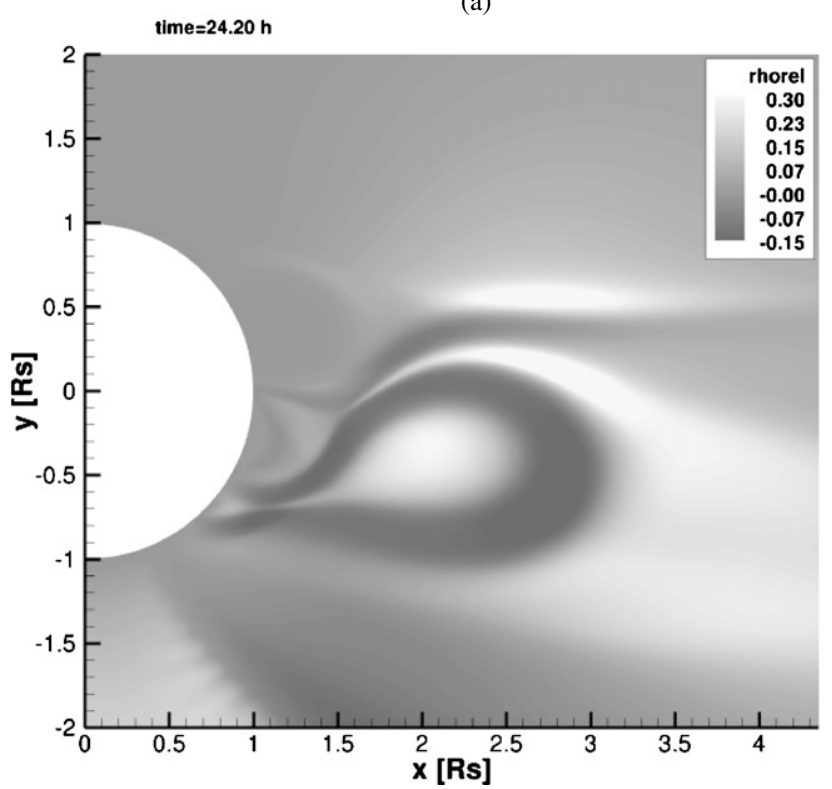

(b)

Figure 13. COR1 image taken at 22:15 UT on 2009 September 21 (a) and (b) snapshot of the relative density for the simulation taken $24.2 \mathrm{hr}$ after the helicity injection began.

to the altitude reconstruction of the prominence. For both the simulated and the observed CME, it takes about $6 \mathrm{hr}$ to reach an altitude of $4 R_{\odot}$ and in both cases the CME is slow.

In order to further compare the dynamics of the simulated and observed CMEs, in Figure 14(b) we show the latitude-time plot for both the simulation (dashed line) and the reconstructed CME ("plus" signs). The prominence has a latitude of about $35^{\circ}$ south and at time $-5 \mathrm{hr}(15: 00$ UT on 2009 September 21) it is evolving in the EUVI-B FOV. In about one and half hours it reaches a latitude of about $31^{\circ}$ south and disappears from the EUVI FOV. The simulated flux rope starts from a location of about $33^{\circ}$ south and experiences a deflection of about $20^{\circ}$ in $3 \mathrm{hr}$, approaching an altitude of $2.25 R_{\odot}$. At this altitude, the core of the observed CME is visible in the COR1 FOV and its latitudinal deflection can be followed for another three hours. At $2.25 R_{\odot}$, the core of the observed CME has a latitude of about

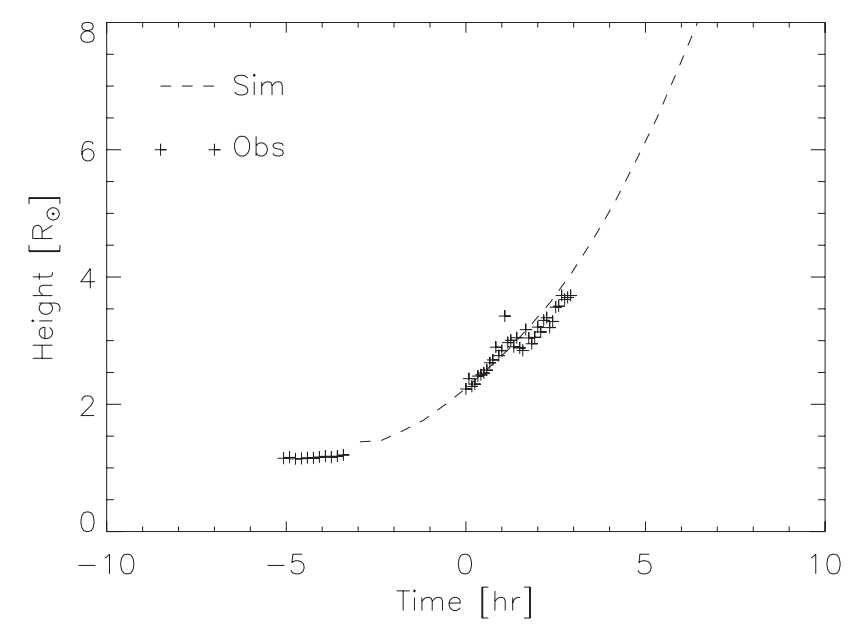

(a)

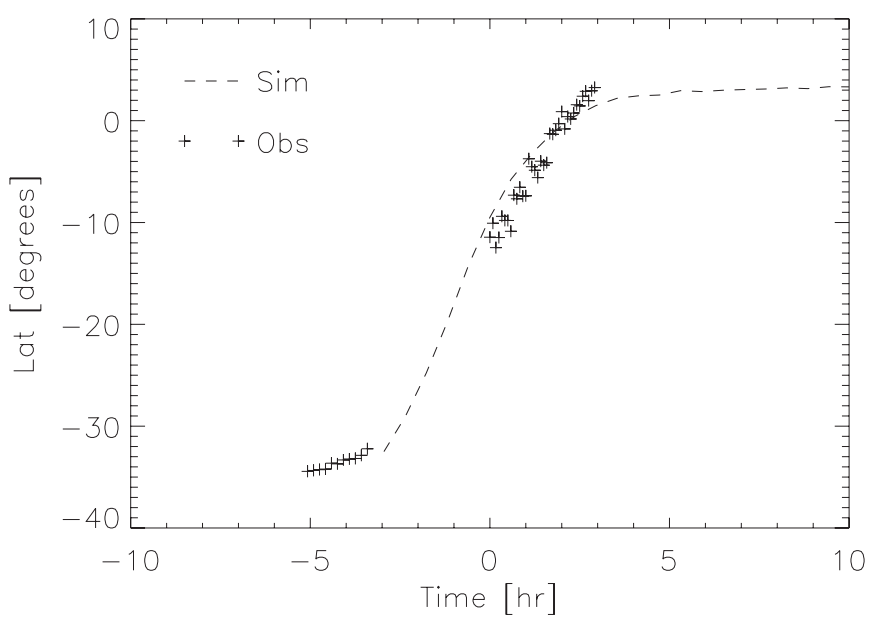

(b)

Figure 14. Comparison between the simulation (dashed line) and observation ("plus" signs). (a) Altitude vs. time and (b) latitude vs. time. Time zero is 20:00 UT on 2009 September 21, corresponding to the time at which the CME was at $2.25 R_{\odot}$.

$12^{\circ}$ south and quickly approaches a latitude of about $4^{\circ}$ north. This latitudinal behavior is well reproduced by the simulation. We have discussed that the deflection of the flux rope is the result of the magnetic pressure unbalance between the southern and the northern part of the flux rope, due to the presence of the $X$-point. However, a key role in the deflection of the CME is also played by the overlying field of the helmet streamer. In order to further investigate the role of the helmet streamer in the deflection of the CME, we performed a numerical simulation in which the strength of the ambient dipole was increased by $33 \%$. As a result of the stronger dipole field, the pseudostreamer structure is no longer present and the AR, together with the northern and southern arcades, is embedded in a large streamer structure, in which the current sheet is tilted slightly north. Figure 15 shows the latitude versus altitude plot for both simulations. In both cases, the two flux ropes originate at a similar latitude, but in the case with a stronger overlying field the center of the flux rope has a smaller altitude. Due to the stronger magnetic tension and pressure forces in the southern part of the expanding arcade, the CME has already approached the solar equator within the first two solar radii. At an altitude of $2.3 R_{\odot}$, the $\mathrm{CME}$ is at a latitude of about $8^{\circ}$ north. Afterward, due to the magnetic tension of the northern helmet streamer field, the CME is deflected toward 
The Astrophysical Journal, 744:66 (14pp), 2012 January 1

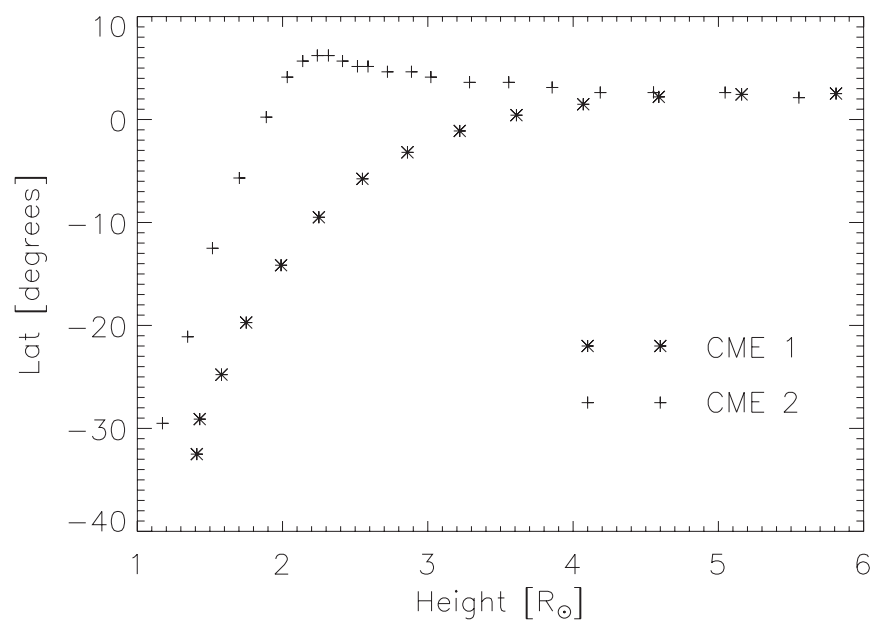

Figure 15. Latitude vs. altitude plot for the simulation discussed in the paper (asterisks) and the simulation with the higher strength of the overlying magnetic field (pluses).

the current sheet undergoing a further deflection and eventually approaching a latitude of about $3^{\circ}$ north. After about $5 R_{\odot}$, both CMEs have reached the HCS and propagate along it.

In Section 2.3, we reconstructed the true trajectory of the $\mathrm{CME}$. Together with the latitudinal and radial profiles, we also discussed the longitudinal trajectory of the CME. We have shown that the CME propagates at an almost constant longitude, resulting in a dynamics that mainly develops along a meridional plane. This result partially justifies the use of $2.5 \mathrm{D}$ simulations for the investigation of this event. Moreover, we have shown that the key dynamical aspects of this CME, such as the trajectory, the structure, and the speed, are well reproduced in the simulation, further confirming the hypothesis that, at least for this event, such axisymmetric simulations are sufficient to quantitatively reproduce and understand the event.

\section{SUMMARY}

Using the two vantage points of the STEREO spacecraft, we have reconstructed the 3D trajectory of the 2009 September 21 CME. We found that, during its propagation in the COR1 FOV, the CME underwent a latitudinal deflection of about $14^{\circ}$. We have also demonstrated that the CME propagated mainly along a meridional plane, showing only minor longitudinal deflections.

In order to simulate the aforementioned event, we performed 2.5D numerical MHD simulations. To simulate the solar corona, we used an initial state that closely resembled the PFSS extrapolations for the Carrington rotation number 2088. To form the flux rope and initiate the event, we applied localized photospheric shearing motions that injected an amount of helicity comparable with what has been measured for this event into the system. In the simulation, the flux rope was formed at a latitude of about $33^{\circ}$ south. As a consequence of the magnetic pressure imbalance due to the interchange reconnection that occurred in the null point inside the pseudostreamer, during its outward propagation the CME was rapidly deflected toward the equator. We compared the altitude-time and latitude-time plots for the simulation with the ones obtained from the reconstructed CME trajectory. We found that the simulation succeeded in reproducing both of them. We also compared our relative density images with the COR 1 images and we find a good agreement with the three-part structure observed by the STEREO-B coronagraph.
In order to investigate the role of the overlying field in the deflection of the CME, we performed a second simulation in which the strength of the ambient field was increased about $33 \%$. As a result of the increased magnetic tension of the overlying field, the CME undergoes a more sudden deflection toward the equator, eventually propagating along the IP current sheet. The increased strength of the overlying field also resulted in a different CME-streamer interaction, the discussion of which will be the focus of a subsequent paper.

To conclude, this study shows that during solar minima, even CMEs originating from high latitude can be easily deflected toward the HCS, eventually resulting in geoeffective events. How rapidly they are deflected depends on the strength of both the overlying magnetic field and the flux rope magnetic flux.

This research was funded by projects GOA-2009-009 (K.U.Leuven), G.0304.07 (FWO Vlaanderen), 3E090665 (FWO Vlaanderen), and C 90347 (ESA Prodex 9). Support from the European Commission's Seventh Framework Programme (FP7-2007-2013) under the grant no. 218816 (SOTERIA project) is gratefully acknowledged. For the simulations, we used the infrastructure of the VSC-Flemish Supercomputer Center, funded by the Hercules foundation and the Flemish Government - department EWI. STEREO is a NASA project. $\mathrm{SOHO}$ is a joint ESA and NASA project.

\section{REFERENCES}

Bemporad, A., Sterling, A. C., Moore, R. L., \& Poletto, G. 2005, ApJ, 635, L189

Berger, M. A., \& Field, G. B. 1984, J. Fluid Mech., 147, 133

Billings, D. E. 1966, A Guide to the Solar Corona (New York: Academic Press) Byrne, J. P., Maloney, S. A., McAteer, R. T. J., et al. 2010, Nat. Commun., 1, 74 Cremades, H., Bothmer, V., \& Tripathi, D. 2006, Adv. Space Res., 38, 461

Dere, K. P., Wang, D., \& Howard, R. 2005, ApJ, 620, L119

Evans, C. R., \& Hawley, J. F. 1988, ApJ, 332, 659

Fengsi, W., \& Dryer, M. 1991, Solar Phys., 132, 373

Filippov, B. P., Gopalswamy, N., \& Lozhechkin, A. V. 2001, Sol. Phys., 203, 119

Gopalswamy, N., Mäkelä, P., Xie, H., Akiyama, S., \& Yashiro, S. 2009, J Geophys. Res. (Space Phys.), 114, 0

Groth, C. P. T., De Zeeuw, D. L., Gombosi, T. I., \& Powell, K. G. 2000, J. Geophys. Res., 105, 25053

Howard, R. F., Harvey, J. W., \& Forgach, S. 1990, Sol. Phys., 130, 295

Inhester, B. 2006, Publ. Int. Space Sci. Inst., arXiv:astro-ph/0612649

Intriligator, D. S., Sun, W., Dryer, M., et al. 2005, J. Geophys. Res. (Space Phys.), 110, 9

Jiang, Y., Yang, J., Zheng, R., Bi, Y., \& Yang, X. 2009, ApJ, 693, 1851

Kaiser, M. L., Kucera, T. A., Davila, J. M., et al. 2008, Space Sci. Rev., 136, 5

Kilpua, E. K. J., Pomoell, J., Vourlidas, A., et al. 2009, Ann. Geophys., 27, 4491

Liewer, P. C., Dejong, E. M., Hall, J. R., et al. 2010, in AIP Conf. Proc. 1216, Twelfth International Solar Wind Conference, ed. M. Maksimovic et al. (Melville, NY: AIP), 412

Lionello, R., Linker, J. A., \& Mikić, Z. 2009, ApJ, 690, 902

Liu, Y., Davies, J. A., Luhmann, J. G., et al. 2010a, ApJ, 710, L82

Liu, Y., Luhmann, J. G., Lin, R. P., et al. 2009, ApJ, 698, L51

Liu, Y., Thernisien, A., Luhmann, J. G., et al. 2010b, ApJ, 722, 1762

Lugaz, N., Hernandez-Charpak, J. N., Roussev, I. I., et al. 2010, ApJ, 715, 493

Lugaz, N., Vourlidas, A., Roussev, I. I., \& Morgan, H. 2009, Sol. Phys., 256, 269

MacQueen, R. M., Hundhausen, A. J., \& Conover, C. W. 1986, J. Geophys. Res., 91, 31

Manchester, W. B., Gombosi, T. I., Roussev, I., et al. 2004, J. Geophys. Res., 109, A02107

Manchester, W. B., IV, Vourlidas, A., Tóth, G., et al. 2008, ApJ, 684, 1448

Mierla, M., Chifu, I., Inhester, B., Rodriguez, L., \& Zhukov, A. 2011, A\&A, 530, L1

Mierla, M., Inhester, B., Antunes, A., et al. 2010, Ann. Geophys., 28, 203

Minnaert, M. 1930, Z. Astrophys., 1, 209

Moore, R. L., \& Sterling, A. C. 2007, ApJ, 661, 543 
Moran, T. G., \& Davila, J. M. 2004, Science, 305, 66

Odstrcil, D., Pizzo, V. J., \& Arge, C. N. 2005, J. Geophys. Res. (Space Phys.), 110,2106

Pariat, E., Démoulin, P., \& Berger, M. A. 2005, A\&A, 439, 1191

Rušin, V., Druckmüller, M., Aniol, P., et al. 2010, A\&A, 513, A45

Schrijver, C. J., \& DeRosa, M. L. 2003, Sol. Phys., 212, 165

Schuck, P. W. 2005, ApJ, 632, L53

Shen, C., Wang, Y., Gui, B., Ye, P., \& Wang, S. 2011, Sol. Phys., 269, 389

Smyrli, A., Zuccarello, F., Romano, P., et al. 2010, A\&A, 521, A56

Srivastava, N., Schwenn, R., Inhester, B., Stenborg, G., \& Podlipnik, B. 1999, in AIP Conf. Ser. 471, The Solar Wind Nine Conference, ed. S. R. Habbal \& C. D. Halas (Melville, NY: AIP), 115
Subramanian, P., Dere, K. P., Rich, N. B., \& Howard, R. A. 1999, J. Geophys. Res., 104, 22321

Thompson, W. T., Davila, J. M., Fisher, R. R., et al. 2003, Proc. SPIE, 4853, 1

Thompson, W. T., \& Reginald, N. L. 2008, Sol. Phys., 250, 443

Tóth, G. 1996, Astrophys. Lett. Commun., 34, 245

van der Holst, B., Jacobs, C., \& Poedts, S. 2007, ApJ, 671, L77

van de Hulst, H. C. 1950, Bull. Astron. Inst. Neth., 11, 135

Wang, Y.-M., Sheeley, N. R., Jr., \& Rich, N. B. 2007, ApJ, 658, 1340

Wei, F., \& Dryer, M. 1991, Solar Phys., 132, 373

Wuelser, J.-P., Lemen, J. R., Tarbell, T. D., et al. 2004, Proc. SPIE, 5171, 111

Zhao, X. P., Plunkett, S. P., \& Liu, W. 2002, J. Geophys. Res., 107, 1223

Zuccarello, F. P., Jacobs, C., Soenen, A., et al. 2009, A\&A, 507, 441 